\title{
Remarks on ergodicity and invariant occupation measure in branching diffusions with immigration ${ }^{\text {th }}$
}

\author{
Reinhard Höpfner ${ }^{\mathrm{a}}$, Eva Löcherbach ${ }^{\mathrm{b}, *}$ \\ ${ }^{a}$ Fachbereich 17 Mathematik und Informatik, Johannes Gutenberg Universität Mainz, 55099 Mainz, Germany \\ ${ }^{\mathrm{b}}$ UFR sciences et technologie, université Paris XII - Val de Marne, 94010 Créteil cedex, France
}

Received 12 March 2004; accepted 24 September 2004

Available online 1 February 2005

\begin{abstract}
We give a necessary and sufficient condition for ergodicity with finite invariant occupation measure for branching diffusions with immigration. We do not assume uniformly subcritial reproduction means. We discuss the structure of the invariant occupation measure and of its density.

@ 2005 Elsevier SAS. All rights reserved.

\section{Résumé}

Nous considérons des diffusions avec branchement et immigration dans $\mathbb{R}^{d}$. Nous donnons une condition nécessaire et suffisante pour que ce processus soit ergodique et sa mesure invariante d'occupation (mesure d'intensité) une mesure finie. Le branchement n'est pas supposé strictement sous-critique. Nous étudions la structure de la mesure invariante d'occupation et (si celle-ci existe) de sa densité de Lebesgue.
\end{abstract}

(c) 2005 Elsevier SAS. All rights reserved.

$M S C: 60 \mathrm{~J} 60 ; 60 \mathrm{~J} 80 ; 62 \mathrm{M} 30$

Keywords: Diffusing particles; Branching; Immigration; Spatial subcriticality; Invariant occupation measure; Invariant occupation density;

Resolvants; Stochastic flows

\footnotetext{
W Work supported in parts by: Deutsche Forschungsgemeinschaft, Schwerpunktprogramm 'Interagierende stochastische Systeme von hoher Komplexität' (SPP-1033), and European Community's Human Potential Programme, under contract HPRN-CT-2000-00100 (DYNSTOCH).

* Corresponding author.

E-mail addresses: hoepfner@mathematik.uni-mainz.de (R. Höpfner), locherbach@ univ-paris12.fr (E. Löcherbach).
} 


\section{Introduction}

This note deals with ergodicity with finite invariant occupation measure in branching diffusions with immigration, and with the properties of a Lebesgue density - when it exists - for the invariant occupation measure.

We consider a particle process where finitely many particles living in $\mathbb{R}^{d}$ move independently of each other on paths which are solutions to SDE's

$$
\mathrm{d} \xi_{s}=b\left(\xi_{s}\right) \mathrm{d} s+\sigma\left(\xi_{s}\right) \mathrm{d} W_{s}
$$

with $m$-dimensional Brownian motions $W$, and undergo branching at random times according to a positiondependent branching rate $\kappa(\cdot)$ and a position-dependent reproduction law $\left(p_{k}(\cdot)\right)_{k \in \mathbb{N}_{0}}$ (a parent particle in position $v \in \mathbb{R}^{d}$ at time $t \geqslant 0$ will die in a small time interval $\left.] t, t+h\right]$ with probability $\kappa(v) h+o(h), h \rightarrow 0$, leaving with probability $p_{k}(v) k$ descendants at $v$ ); in addition, there are immigration events occurring at constant rate $c$ where one new particle is added to the pre-existing configuration in a position chosen according to a fixed probability law $\pi$.

This describes a strong Markov process $\eta=\left(\eta_{t}\right)_{t}$ of (ordered) finite particle configurations, called a branching diffusion with immigration. Its configuration state space $S$ is given by

$$
S=\bigcup_{l=0}^{\infty}\left(\mathbb{R}^{d}\right)^{l}
$$

which consists of all (ordered) configurations $x=\left(x^{1}, \ldots, x^{l}\right), x^{i} \in \mathbb{R}^{d}, 1 \leqslant i \leqslant l, l \geqslant 1$, with $\left(\mathbb{R}^{d}\right)^{0}=\{\Delta\}$, and which is a Polish space. The length of a configuration $x \in S$ is denoted by $l(x)$. Sometimes we write a configuration $x \in S$ as a point measure on $\mathbb{R}^{d}: x(A)=\sum_{i=1}^{l(x)} \delta_{x^{i}}(A)$ if $l(x) \geqslant 1$, and $\Delta(A)=0$.

As a special case of the construction in Löcherbach [14], we construct the branching diffusion with immigration as a càdlàg process $\eta=\left(\eta_{t}\right)_{0 \leqslant t<\zeta}$ with lifetime $\zeta$ (due to possible explosion of the process) (cf. Dellacherie and Meyer [3, XIV,23-24]), whose jumps correspond to either branching or immigration events. Arranging the sequence $\left(T_{n}\right)_{n}$ of branching or immigration times in increasing order, with $T_{0} \equiv 0$ and $T_{n} \uparrow \zeta$, the process $\eta$ is characterized by the following assertions $\mathrm{A} 1+\mathrm{A} 2$ :

(A1) In restriction to every random interval $\llbracket T_{n}, T_{n+1} \llbracket, n \geqslant 0$ : Writing $l$ for the length of the configuration $\eta_{T_{n}}$, $\left(\eta_{T_{n}+s}\right)_{s}$ is the motion of $l$ independent particles according to (1), stopped at configuration dependent rate

$$
\alpha\left(x^{1}, \ldots, x^{l}\right)=c+\sum_{i=1}^{l} \kappa\left(x^{i}\right) \quad \text { if } x=\left(x^{1}, \ldots, x^{l}\right) \text { with } l \geqslant 1, \quad \alpha(\Delta)=c
$$

where $\Delta$ denotes the void configuration. Thus

(i) in case $\eta_{T_{n}} \neq \Delta$ : with starting point $\left(x^{1}, \ldots, x^{l}\right):=\eta_{T_{n}}$, the $l$-particle motion after time $T_{n}$ evolves as $\left(\xi^{1}, \ldots, \xi^{l}\right)$ solution of

$$
\mathrm{d} \xi_{s}^{i}=b\left(\xi_{s}^{i}\right) \mathrm{d} s+\sigma\left(\xi_{s}^{i}\right) \mathrm{d} W_{s}^{i}, \quad 1 \leqslant i \leqslant l,
$$

with independent Brownian motions $W^{1}, \ldots, W^{l}$, and conditionally on evolution of $\left(\xi^{1}, \ldots, \xi^{l}\right)$ the probability to have $T_{n+1}-T_{n}>v$ is $\exp \left\{-\int_{0}^{v} \mathrm{~d} s \alpha\left(\xi_{s}^{1}, \ldots, \xi_{s}^{l}\right)\right\}, 0<v<\infty$;

(ii) in case $\eta_{T_{n}}=\Delta$ : the trajectory $\left(\eta_{T_{n}+s}\right)_{S}$ is the constant function $\Delta$, up to time $T_{n+1}-T_{n}$ which is an independent exponential time with parameter $c$. 
(A2) At jump times $T_{n+1}, n \geqslant 0$ : the transition from $\eta_{T_{n+1}^{-}}$to $\eta_{T_{n+1}}$ is governed by a transition probability $K(\cdot, \cdot)$ on the configuration space $S$ (see (2) above): for $x=\left(x^{1}, \ldots, x^{l}\right)$ with $l \in \mathbb{N}_{0}$ (this means $x=\Delta$ if $l=0$ ), $K(x, \cdot)$ is the law

$$
K(x, \cdot)=\sum_{i=1}^{l} \frac{\kappa\left(x^{i}\right)}{\alpha(x)}\left(\sum_{k \in \mathbb{N}_{0}} p_{k}\left(x^{i}\right) \delta_{\left(\Pi_{l, i, k}(x)\right)}\right)+\frac{c}{\alpha(x)} \int_{\mathbb{R}^{d}} \pi(\mathrm{d} v) \delta_{\left(x^{1}, \ldots, x^{l}, v\right)}
$$

where $\Pi_{l, i, k}(x)$ is the configuration $(x^{1}, \ldots, x^{i-1}, x^{i+1}, \ldots, x^{l}, \underbrace{x^{i}, \ldots, x^{i}}_{k \text { times }})$ obtained from $x=\left(x^{1}, \ldots, x^{l}\right)$ by death of the $i$-th particle with $k$ offspring at the death position.

In contrast to the construction in Löcherbach [14], we do not require $k \neq 1$ offspring in the reproduction laws, and we are not primarily interested in the construction of $\eta$ as canonical process on a canonical path space (where jumps with $k=1$ offspring may be unobservable, which is not convenient for purposes of statistical inference); also we do not rearrange the particles at random at every jump time $T_{n}$.

We wish to have simple conditions in terms of $b(\cdot), \sigma(\cdot), \kappa(\cdot),\left(p_{k}(\cdot)\right)_{k}, \pi$ which imply the following properties $\mathrm{P} 1+\mathrm{P} 2+\mathrm{P} 3$ of the process $\eta$ :

P1: No accumulation of jumps in finite time intervals, thus in particular $\zeta=+\infty$ a.s.

P2: Ergodicity, i.e. we wish the process $\eta=\left(\eta_{t}\right)_{t \geqslant 0}$ to be recurrent in the sense of Harris, admitting $\Delta$ as recurrent atom, and such that the invariant measure $m$ on $S$

$$
m(F)=E_{\Delta}\left(\int_{0}^{R} \mathrm{~d} s 1_{F}\left(\eta_{s}\right)\right), \quad F \in \mathcal{B}(\mathcal{S})
$$

is a finite measure. Here $R:=\inf \left\{T_{n}: n \geqslant 1, \eta_{T_{n}}=\Delta\right\}$ is the time of first return to the void configuration $\Delta$. We do not normalize the total mass of $m$ to 1 .

P3: Finite invariant occupation measure: associating to $m$ the measure

$$
\bar{m}(A)=\int_{S} m(\mathrm{~d} x) x(A)=E_{\Delta}\left(\int_{0}^{R} \mathrm{~d} s \eta_{s}(A)\right), \quad A \in \mathcal{B}\left(\mathbb{R}^{d}\right),
$$

we wish to have

$$
\bar{m}\left(\mathbb{R}^{d}\right)=\int_{S} m(\mathrm{~d} x) l(x)<\infty .
$$

We call $\bar{m}$ the invariant occupation measure, or the intensity of $m$, sometimes also invariant measure on $\mathbb{R}^{d}$.

We shall give - under additional assumptions on the quantities determining the process (see Assumptions 1.1 and 1.3 below) - a simple necessary and sufficient condition for properties $\mathrm{P} 1+\mathrm{P} 2+\mathrm{P} 3$. Under this condition, we shall derive simple closed form expressions for the measure $\bar{m}$ on $\mathbb{R}^{d}$ and - under stronger conditions - for its Lebesgue density (whenever this density exists).

In the context of statistical inference, where an ergodic branching diffusion with immigration is observed over a long time interval, with drift, branching rates etc. either depending on an unknown parameter, or belonging to certain function classes, we need to be able 
(i) to check properties $\mathrm{P} 1+\mathrm{P} 2+\mathrm{P} 3$ for the model in question, and

(ii) to know invariant occupation measures and densities explicitly.

Among conditions needed for local asymptotic normality (LAN) in branching diffusion with immigration, see Le Cam and Yang [13] or Ibragimov and Has'minskii [8] for a general statistical background, the essential condition is integrability of certain information functionals with respect to the invariant measure, see Löcherbach [15]. In nonparametric problems where e.g. the branching rate is considered as an unknown function to be estimated e.g. by kernel estimates, nonparametric rates of convergence depend on smoothness classes for the invariant occupation density, similar to the classical iid density estimation problem, see Höpfner, Hoffmann and Löcherbach [6].

In dimension $d=1$, local time and Tanaka's formula can be used to get an invariant occupation density, but this requires moment conditions with respect to the invariant occupation measure. There is no analogue to this approach in higher dimensions. In arbitrary dimension $d \geqslant 1$, once the invariant occupation measure is identified as a certain resolvant related to the one-particle motion, results from Malliavin calculus can be used to obtain $\mathcal{C}^{\infty}$-smoothness of the invariant occupation density, see Cattiaux [2]. This approach needs strong conditions (drift and diffusion coefficient of (1) are $\mathcal{C}_{b}^{\infty}$, and the mass reduction rate is $\mathcal{C}_{b}^{\infty}$ and bounded away from 0 ). It can be extended to particle processes with interaction, see Löcherbach [16]. However, conditions of type $\mathcal{C}_{b}^{\infty}$ are restrictive and sometimes undesirable; already the simplest models - e.g., constant mass reduction rate and particles moving on Ornstein-Uhlenbeck paths - are ruled out.

The aim of this note is to give a self-contained investigation of ergodicity of branching diffusions with immigration, and of invariant occupation measure and density, in an arbitrary dimension $d \geqslant 1$ under minimal conditions (Theorems 1.6 and 1.7, Theorem 3.5 and Lemma 3.6); the result under $\mathcal{C}_{b}^{\infty}$-conditions appears in Theorem 3.9. The results are stated in Sections 1 and 3, the proofs are collected in Sections 2 and 4.

\section{Ergodicity with finite invariant occupation measure}

We specify the assumptions which we impose on the quantities determining the process $\left(\eta_{t}\right)_{t}$. Write $\mathcal{C}_{(b)}^{k}\left(\mathbb{R}^{p}, \mathbb{R}^{q}\right)$ for the space of $\mathcal{C}^{k}$-functions $\mathbb{R}^{p} \rightarrow \mathbb{R}^{q}$ for which all partial derivatives of orders $1, \ldots, k$ are bounded. $\mathcal{C}_{b}^{k}$ then denotes the subspace of bounded functions in $\mathcal{C}_{(b)}^{k}$, and $\mathcal{C}_{b}$ is $\mathcal{C}_{b}^{0}$; subscript $\mathcal{K}$ denotes compact support.

All proofs for the results stated in Section 1 will be given in Section 2.

\subsection{Assumptions.}

(a) Drift and diffusion coefficient of the diffusion $\xi$ in (1): we assume that $b: \mathbb{R}^{d} \rightarrow \mathbb{R}^{d}$ and $\sigma: \mathbb{R}^{d} \rightarrow \mathbb{R}^{d \times m}$ are globally Lipschitz continuous, and write $a:=\sigma \sigma^{\top}$.

(b) Branching rate: $\kappa \in \mathcal{C}_{b}\left(\mathbb{R}^{d}, \mathbb{R}\right)$ is strictly positive, and

$$
\int_{0}^{\infty} \mathrm{d} s \kappa\left(\xi_{s}\right)=\infty \quad \text { a.s. }
$$

for every choice of a starting point $v \in \mathbb{R}^{d}$ for the diffusion (1).

(c) Reproduction laws: with $\mathcal{M}^{1}\left(\mathbb{N}_{0}\right)$ denoting the space of all probability measures on $\mathbb{N}_{0}$ and $p=\left(p_{k}(\cdot)\right)_{k}$, the mapping $p: \mathbb{R}^{d} \rightarrow \mathcal{M}^{1}\left(\mathbb{N}_{0}\right)$ is continuous with

$$
\rho \in \mathcal{C}_{b}\left(\mathbb{R}^{d}, \mathbb{R}\right), \quad \rho(v):=\sum_{k=0}^{\infty} k p_{k}(v), \quad v \in \mathbb{R}^{d} .
$$


1.2. Remarks. Note that we do not assume that the function $\rho(\cdot)$ is $[0,1)$-valued.

The mass reduction (or augmentation) rate $[\kappa(1-\rho)](\cdot)$ is by 1.1 a bounded function on $\mathbb{R}^{d}$. Note also that we did not make any assumption on the immigration law: with $\mathcal{M}^{1}\left(\mathbb{R}^{d}\right)$ the space of all probability measures on $\mathbb{R}^{d}$, we allow for any $\pi \in \mathcal{M}^{1}\left(\mathbb{R}^{d}\right)$.

We may have $b=0$ or $\sigma=0$ on certain subsets of $\mathbb{R}^{d}$.

1.3. Assumption ('Spatial subcriticality'). Write $T$ for the killing time of a particle travelling on the path of $\xi$ under position-dependent killing at rate $\kappa(\cdot)(T$ is a.s. finite by 1.1(b)). We assume that in the class of all kernels $H(\cdot, \cdot)$ on $\left(\mathbb{R}^{d}, \mathcal{B}\left(\mathbb{R}^{d}\right)\right)$, there is a finite kernel solving

$$
H(v, f)=E_{v}\left(\int_{0}^{T} f\left(\xi_{s}\right) \mathrm{d} s+\rho\left(\xi_{T}\right) H\left(\xi_{T}, f\right)\right), \quad f \in \mathcal{C}_{b}\left(\mathbb{R}^{d}, \mathbb{R}^{+}\right), v \in \mathbb{R}^{d} .
$$

In our note, 'kernel' is understood as in Revuz [17, Definition I.1.1] except that we always have $H(v, A) \in$ $[0, \infty]$ for all $v \in \mathbb{R}^{d}, A \in \mathcal{B}\left(\mathbb{R}^{d}\right)$. A finite kernel has $H\left(v, \mathbb{R}^{d}\right)<\infty, v \in \mathbb{R}^{d}$.

1.4. Lemma. Write for short $\gamma:=[\kappa(1-\rho)]$ which is in $\mathcal{C}_{b}\left(\mathbb{R}^{d}, \mathbb{R}\right)$.

(a) Under 1.1, the $\gamma$-resolvent kernel of the diffusion $\xi$

$$
\gamma_{R}(v, f):=E_{v}\left(\int_{0}^{\infty} \mathrm{d} t f\left(\xi_{t}\right) \mathrm{e}^{-\int_{0}^{t} \mathrm{~d} s \gamma\left(\xi_{s}\right)}\right), \quad f \in \mathcal{C}_{b}\left(\mathbb{R}^{d}, \mathbb{R}^{+}\right), v \in \mathbb{R}^{d}
$$

is the (unique) minimal solution to (5).

(b) Under 1.1, Assumption 1.3 (spatial subcriticality) is equivalent to the following condition (6):

$$
E_{v}\left(\int_{0}^{\infty} \mathrm{d} t \mathrm{e}^{-\int_{0}^{t} \mathrm{~d} s \gamma\left(\xi_{s}\right)}\right)<\infty \quad \text { for all } v \in \mathbb{R}^{d} .
$$

\subsection{Remarks.}

(a) We shall see in 2.1 and 2.2 below that Assumption 1.3 is indeed a proper 'spatial' analogue of the classical subcriticality of continuous-time branching processes without immigration.

(b) In view of 1.4(b), an obvious sufficient condition for spatial subcriticality 1.3 is

$$
\inf _{v \in \mathbb{R}^{d}}[\kappa(1-\rho)](v)>0 .
$$

(c) From (6) we see that 1.3 is not satisfied if $\kappa$ and $\rho$ are spatially constant, and $\rho \geqslant 1$ : in this case, the minimal solution of (5) is the trivial one $H(v, A) \equiv+\infty$.

\subsection{Theorem. Assume 1.1 and 1.3 .}

(a) For immigration laws $\pi \in \mathcal{M}^{1}\left(\mathbb{R}^{d}\right)$ satisfying

$$
\pi^{\gamma} R \text { is a finite measure on } \mathbb{R}^{d}
$$

the branching diffusion with immigration $\eta$ has the properties $\mathrm{P} 1+\mathrm{P} 2+\mathrm{P} 3$. 
(b) Under (8), the invariant intensity measure $\bar{m}$ of (4) is given by

$$
\bar{m}(A)=C \pi^{\gamma} R(A), \quad A \in \mathcal{B}\left(\mathbb{R}^{d}\right)
$$

with constant $C$ defined as $c E_{\Delta}(R), c f$. (3).

(c) The branching diffusion with immigration $\eta$ has the properties $\mathrm{P} 1+\mathrm{P} 2+\mathrm{P} 3$ for arbitrary choice of an immigration law $\pi \in \mathcal{M}^{1}\left(\mathbb{R}^{d}\right)$ if and only if the total mass of the resolvant

$$
v \longrightarrow{ }^{\gamma} R\left(v, \mathbb{R}^{d}\right)=E_{v}\left(\int_{0}^{\infty} \mathrm{d} t \mathrm{e}^{-\int_{0}^{t} \mathrm{~d} s \gamma\left(\xi_{s}\right)}\right)
$$

(cf. (6)) is a bounded function on $\mathbb{R}^{d}$.

1.7. Theorem. Assume 1.1 and 1.3 , and let $\sigma(\cdot)$ be bounded on $\mathbb{R}^{d}$. Consider an immigration law $\pi$ such that (8) holds. Then the invariant occupation measure $\bar{m}$ is such that

$$
\text { Af } \in L^{1}(\bar{m}) \text { for all } f \in \mathcal{C}_{b}^{2}:=\mathcal{C}_{b}^{2}\left(\mathbb{R}^{d}, \mathbb{R}\right),
$$

and $\bar{m}$ is a solution to

$$
\bar{m}(A f-\gamma f)=-C \pi(f), \quad f \in \mathcal{C}_{b}^{2},
$$

where $A$ is the Markov generator of the diffusion $\xi$ of (1).

We mention some examples illustrating that the invariant occupation measure in Theorem 1.6, even under the strong ergodicity condition in 1.6(c), will be in general quite far from the usual picture of 'nice' invariant laws (e.g. invariant distributions of one-dimensional ergodic diffusions). The problem of a density for the invariant occupation measure will be considered further in Section 3.

1.8. Examples. Take constant mass reduction rate $\gamma(\cdot) \equiv \gamma>0$ on $\mathbb{R}^{d}$. By $1.6(\mathrm{c})$, we have $\mathrm{P} 1+\mathrm{P} 2+\mathrm{P} 3$ for arbitrary choice of an immigration law $\pi$. Consider the invariant occupation measure $\bar{m}=C \pi^{\gamma} R$, and write $\lambda$ for the Lebesgue measure on $\mathbb{R}^{d}$.

Under the conditions which we have made up to now,

(a) $\bar{m}$ cannot be expected to be $\lambda$-absolutely continuous: 1.1 and 1.3 allow for non-empty interior $U$ of $\left\{v \in \mathbb{R}^{d}: b(v)=0, \sigma(v)=0\right\}$; with choice $\pi:=\delta_{a}$ for some $a \in U$, we obtain $\pi^{\gamma} R=\frac{1}{\gamma} \delta_{a}$.

(b) If a density $\mathrm{d} \bar{m} / \mathrm{d} \lambda$ exists, it cannot be expected to be continuous on $\mathbb{R}^{d}$ : Take $\xi$ in (1) as $d$-dimensional standard Brownian motion, take $d \geqslant 3$. Then ${ }^{\gamma} R(v, d u)$ has Lebesgue density

$$
u \rightarrow \int_{0}^{\infty} \mathrm{d} t \mathrm{e}^{-\gamma t}(2 \pi t)^{-\frac{d}{2}} \mathrm{e}^{-\frac{1}{2}(u-v)^{\top} \frac{1}{t}(u-v)}
$$

which is smooth on $\mathbb{R}^{d} \backslash\{v\}$, and has a singularity at $u=v$ (this is the prototype example for general diffusions with $a(\cdot)=\left(\sigma \sigma^{\top}\right)(\cdot)$ nondegenerate, see Cattiaux [2, Proposition (1.37)]).

(i) The function in (11) is - up to the constant $C$ - the density $\mathrm{d} \bar{m} / \mathrm{d} \lambda$ in case $\pi:=\delta_{v}, v \in \mathbb{R}^{d}$.

(ii) Defining $\pi$ as image of the one-dimensional standard normal law $\mathcal{N}(0,1)$ under the mapping $\mathbb{R} \ni y \rightarrow$ $(y, \ldots, y)=: v \in \mathbb{R}^{d}$ (hence the immigration measure $\pi$ on $\left(\mathbb{R}^{d}, \mathcal{B}\left(\mathbb{R}^{d}\right)\right)$ is concentrated on the diagonal in $\mathbb{R}^{d}$ ), one has from (11) an explicit expression for the Lebesgue density of $\pi^{\gamma} R$. It is easy to see that this density takes the value $+\infty$ at every point $u$ belonging to the diagonal in $\mathbb{R}^{d}$, and is smooth outside the diagonal. 


\section{Proofs for Section 1}

We start with the proof of Lemma 1.4.

2.1. Proof of Lemma 1.4. Assume 1.1 , write $\mathcal{C}_{\mathcal{K}}^{+}:=\mathcal{C}_{\mathcal{K}}\left(\mathbb{R}^{d}, \mathbb{R}^{+}\right)$, and $\gamma:=[\kappa(1-\rho)]$. We have to prove that the $\gamma$-resolvent kernel for the diffusion $\xi$ of (1)

$$
\gamma_{R}(v, f):=E_{v}\left(\int_{0}^{\infty} \mathrm{d} t f\left(\xi_{t}\right) \mathrm{e}^{-\int_{0}^{t} \mathrm{~d} s \gamma\left(\xi_{s}\right)}\right), \quad f \in \mathcal{C}_{\mathcal{K}}^{+}, v \in \mathbb{R}^{d}
$$

on $\left(\mathbb{R}^{d}, \mathcal{B}\left(\mathbb{R}^{d}\right)\right.$ ) (in general not $\sigma$-finite, under 1.1 alone) always solves the problem (5)

$$
H(v, f)=E_{v}\left(\int_{0}^{T} f\left(\xi_{s}\right) \mathrm{d} s+\rho\left(\xi_{T}\right) H\left(\xi_{T}, f\right)\right), \quad f \in \mathcal{C}_{\mathcal{K}}^{+}, v \in \mathbb{R}^{d},
$$

and is in fact the minimal solution of (5).

Thus we prove assertion (a) of Lemma 1.4; (b) is an immediate consequence.

1. The total expected occupation time of the diffusion $\xi$ starting at $v$ and killed at rate $\kappa(\cdot)$ is

$$
{ }^{\kappa} U(v, A):=E_{v}\left(\int_{0}^{\infty} \mathrm{d} t 1_{A}\left(\xi_{t}\right) \mathrm{e}^{-\int_{0}^{t} \mathrm{~d} s \kappa\left(\xi_{s}\right)}\right) \leqslant \infty, \quad A \in \mathcal{B}\left(\mathbb{R}^{d}\right) .
$$

Note that by Assumption 1.1(b), for all choices of a starting point $v \in \mathbb{R}^{d}$, the diffusion $\xi$ killed at rate $\kappa(\cdot)$ has a.s. a strictly positive and finite lifetime (which obviously does not imply that the expectation in (12) is finite). Multiplying both sides of (12) with $\kappa(\cdot)$ as a density for the second argument, we write $\left[{ }^{\kappa} U \kappa\right]\left(v, \mathrm{~d} v^{\prime}\right):={ }^{\kappa} U\left(v, \mathrm{~d} v^{\prime}\right) \kappa\left(v^{\prime}\right)$; then $\left[{ }^{\kappa} U \kappa\right](\cdot, \cdot)$ is the transition probability

$$
\left[{ }^{\kappa} U \kappa\right](v, A)=E_{v}\left(\int_{0}^{\infty} \mathrm{d} t 1_{A}\left(\xi_{t}\right) \kappa\left(\xi_{t}\right) \mathrm{e}^{-\int_{0}^{t} \mathrm{~d} s \kappa\left(\xi_{s}\right)}\right)
$$

on $\left(\mathbb{R}^{d}, \mathcal{B}\left(\mathbb{R}^{d}\right)\right)$ which selects the killing position of the particle to be killed at rate $\kappa(\cdot)$ on the path of $\xi$. Write $T$ for this killing time. Then for $f, g \in \mathcal{C}_{\mathcal{K}}^{+}$

$$
E_{v}\left(\left[\frac{1}{\kappa} f\right]\left(\xi_{T}\right)\right)={ }^{\kappa} U(v, f)=E_{v}\left(\int_{0}^{T} \mathrm{~d} t f\left(\xi_{t}\right)\right)
$$

so the problem (5) takes the form

$$
H(v, f)={ }^{\kappa} U(v, f)+\int_{\mathbb{R}^{d}}\left[{ }^{\kappa} U \kappa\right]\left(v, d v^{\prime}\right) \rho\left(v^{\prime}\right) H\left(v^{\prime}, f\right), \quad f \in \mathcal{C}_{\mathcal{K}}^{+}, v \in \mathbb{R}^{d}
$$

or multiplied with $\kappa(\cdot)$ as density relative to the second argument

$$
[H \kappa](v, f)=\left[{ }^{\kappa} U \kappa\right](v, f)+\int_{\mathbb{R}^{d}}\left[{ }^{\kappa} U \kappa\right]\left(v, \mathrm{~d} v^{\prime}\right) \rho\left(v^{\prime}\right)[H \kappa]\left(v^{\prime}, f\right), \quad f \in \mathcal{C}_{\mathcal{K}}^{+}, v \in \mathbb{R}^{d} .
$$

2. We discuss the class of all solutions $[H \kappa]$ to (14). Iterating (14), we get for every $N$ fixed

$$
[H \kappa]=\left(\sum_{n=0}^{N}\left(\left[{ }^{\kappa} U \kappa\right] \rho\right)^{n}\right)\left[{ }^{\kappa} U \kappa\right]+\left(\left[{ }^{\kappa} U \kappa\right] \rho\right)^{N+1}[H \kappa] .
$$


Note that the second term on the r.h.s of (15) is necessarily decreasing in $N$ (the first term of the r.h.s. is increasing in $N$ and the 1.h.s. does not depend on $N$ ), for arguments $v \in \mathbb{R}^{d}, f \in \mathcal{C}_{\mathcal{K}}^{+}$, so all solutions to (14) are of the form

$$
[H \kappa]=\left(\sum_{n=0}^{\infty}\left(\left[{ }^{\kappa} U \kappa\right] \rho\right)^{n}\right)\left[{ }^{\kappa} U \kappa\right]+J, \quad J:=\lim _{N \rightarrow \infty}\left(\left[{ }^{\kappa} U \kappa\right] \rho\right)^{N+1}[H \kappa]
$$

where $J(\cdot, \cdot)$ by $(14)$ is a solution to

$$
J(v, f) \geqslant 0, \quad J(v, f)=E_{v}\left(\rho\left(\xi_{T}\right) J\left(\xi_{T}, f\right)\right), \quad v \in \mathbb{R}^{d}, f \in \mathcal{C}_{\mathcal{K}}^{+} .
$$

Note that $[H \kappa](v, f) \equiv \infty$ is always a solution to (14), so we may have $J(v, f) \equiv \infty$.

We shall prove in step 3 below that

$$
\left(\sum_{n=0}^{\infty}\left(\left[{ }^{\kappa} U \kappa\right] \rho\right)^{n}\right)\left[{ }^{\kappa} U \kappa\right]
$$

equals (without conditions other that 1.1) the resolvent kernel ${ }^{\gamma} R(\cdot, \cdot)$ multiplied with $\kappa$ as a density for the second argument. Hence (18) is always a kernel. Since the kernel (18) solves (14), it is by (16) + (17) the minimal solution of (14).

Hence ${ }^{\gamma} R(\cdot, \cdot)$ is the (unique) minimal solution to (13), i.e. to (5).

3. We calculate the kernel in (18). First, we express $\left[{ }^{\kappa} U \kappa\right](\cdot, \cdot)$ in terms of the law of some diffusion $\tilde{\xi}$ observed after an independent exponential time. Write $A$ for the strictly increasing additive functional $A_{t}:=\int_{0}^{t} \kappa\left(\xi_{s}\right) \mathrm{d} s$ of $\xi$, and $\tau$ for its inverse:

$$
\tau_{r}=\inf \left\{t: A_{t}>r\right\}, \quad \tau(\mathrm{d} r)=\frac{1}{\kappa\left(\xi_{\tau_{r}}\right)} \mathrm{d} r .
$$

By $1.1(\mathrm{~b})$, this is a.s. a time change $[0, \infty) \leftrightarrow[0, \infty)$, for every choice of the starting point $v$. Then the time changed diffusion

$$
\tilde{\xi}_{r}:=\xi_{\tau_{r}}, \quad r \geqslant 0,
$$

satisfies the equation

$$
\mathrm{d} \tilde{\xi}_{r}=\tilde{b}\left(\tilde{\xi}_{r}\right) \mathrm{d} r+\tilde{\sigma}\left(\tilde{\xi}_{r}\right) \mathrm{d} \tilde{W}_{r}, \quad \tilde{b}=\frac{b}{\kappa}, \tilde{\sigma}=\frac{\sigma}{\sqrt{\kappa}},
$$

and we have for $f \in \mathcal{C}_{\mathcal{K}}$

$$
\begin{aligned}
{\left[{ }^{\kappa} U \kappa\right](v, f) } & =E_{v}\left(\int_{0}^{\infty} \mathrm{d} t f\left(\xi_{t}\right) \kappa\left(\xi_{t}\right) \mathrm{e}^{-\int_{0}^{t} \mathrm{~d} s \kappa\left(\xi_{s}\right)}\right)=E_{v}\left(\int_{0}^{\infty} \tau(\mathrm{d} r) f\left(\xi_{\tau_{r}}\right) \kappa\left(\xi_{\tau_{r}}\right) \mathrm{e}^{-r}\right) \\
& =E_{v}\left(\int_{0}^{\infty} \mathrm{d} r \mathrm{e}^{-r} f\left(\tilde{\xi}_{r}\right)\right)=\widetilde{R}(v, f)
\end{aligned}
$$

where $\widetilde{R}=\int_{0}^{\infty} \mathrm{d} t \mathrm{e}^{-t} \widetilde{P}_{t}$ is the resolvent kernel for the diffusion $\tilde{\xi}$. Since $\left[{ }^{\kappa} U \kappa\right]=\widetilde{R}$, Eq. (14) takes the form

$$
[H \kappa](v, f)=E_{v}\left(f\left(\tilde{\xi}_{S_{1}}\right)+\rho\left(\tilde{\xi}_{S_{1}}\right)[H \kappa]\left(\tilde{\xi}_{S_{1}}, f\right)\right)
$$

where $S_{1}$ is an exponentially distributed time with parameter 1 , independent of the diffusion $\tilde{\xi}$. Preparing independent exponential waiting times

$$
S_{1}, S_{2}-S_{1}, \ldots, S_{n}-S_{n-1}, \ldots
$$


independent of $\tilde{\xi}$, the kernel (18) is (with $\prod_{i=1}^{0}$ defined as 1)

$$
(v, f) \longrightarrow E_{v}\left(\sum_{n=1}^{\infty}\left(\rho\left(\tilde{\xi}_{S_{1}}\right) \cdots \rho\left(\tilde{\xi}_{S_{n-1}}\right)\right) f\left(\tilde{\xi}_{S_{n}}\right)\right) .
$$

Since $\rho(\cdot): \mathbb{R}^{d} \rightarrow[0, \infty)$ is bounded on $\mathbb{R}^{d}$ by 1.1 , the r.h.s of (20) can be transformed exactly as in Höpfner and Löcherbach [5, proof of (5.29), p. 59] into

$$
E_{v}\left(\int_{0}^{\infty} \mathrm{d} t f\left(\tilde{\xi}_{t}\right) \mathrm{e}^{-\int_{0}^{t} \mathrm{~d} s(1-\rho)\left(\tilde{\xi}_{s}\right)}\right)
$$

Changing time back according to step 3 , this is equal to

$$
E_{v}\left(\int_{0}^{\infty} \mathrm{d} t \kappa\left(\xi_{t}\right) f\left(\xi_{t}\right) \mathrm{e}^{-\int_{0}^{t} \mathrm{~d} s[\kappa(1-\rho)]\left(\xi_{s}\right)}\right)=\left[{ }^{\gamma} R \kappa\right](v, f) .
$$

By $(18)+(20)+(22)$, we have proved the assertions at the end of step 2.

This concludes the proof of Lemma 1.3.

We will use the notation $\phi$ to denote subprocesses of the full process $\eta$ called 'subfamilies': with a particle living at a certain time these subprocesses contain the full direct descendance of this particle, and there is no immigration. By the independence assumptions characterizing branching diffusions, the subfamilies are again strongly Markov, and are branching diffusions without immigration.

They take the value $\Delta$ once the last member of the subfamily has died; we write $R^{\phi}$ (which may take the value $+\infty$ ) for the extinction time (that is, the first return to $\Delta$ ) of the subprocess $\phi$.

Subprocesses $\phi$ might have an accumulation of jumps in finite time, and thus finite lifetime $\zeta^{\phi}$ (in the sense of explosion time): we define $\phi:=\Delta$ on $\llbracket \zeta^{\phi}, \infty \llbracket$.

With these conventions, for $s \geqslant 0$ and $v \in \mathbb{R}^{d}, \phi^{(s, v)}=\left(\phi_{t}^{(s, v)}\right)_{t \geqslant s}$ is the subfamily of descendants of a particle which was in $v$ at time $s$. If $\left(T_{j}^{I}, \zeta_{j}^{I}\right)_{j}$ denotes the point process of immigration times/positions in the branching diffusion with immigration $\left(\eta_{t}\right)_{0 \leqslant t<\zeta}$, with immigration times arranged in increasing order, the descendance stemming from the $j$-th immigrant is $\phi^{\left(T_{j}^{I}, \zeta_{j}^{I}\right)}$.

2.2. Proposition. Under 1.1, total expected occupation times for subprocesses $\phi^{(0, v)}$

$$
V(v, A):=E\left(\int_{0}^{\infty} \mathrm{d} t \phi_{t}^{(0, v)}(A)\right), \quad A \in \mathcal{B}\left(\mathbb{R}^{d}\right), v \in \mathbb{R}^{d}
$$

(all integrals under the expectation sign are well defined since $\phi \equiv \Delta$ on $\llbracket \zeta^{\phi}, \infty \llbracket$, and take values in $[0, \infty]$ ) are given by the minimal solution to (5) as specified in Lemma 1.4

$$
V(v, A)={ }^{\gamma} R(v, A)=E_{v}\left(\int_{0}^{\infty} \mathrm{d} t 1_{A}\left(\xi_{t}\right) \mathrm{e}^{-\int_{0}^{t} \mathrm{~d} s[\kappa(1-\rho)]\left(\xi_{s}\right)}\right) .
$$

Proof. Write $\phi^{(0, v) ; N}$ for the subprocess of $\phi^{(0, v)}$ from which all particles belonging to generations later than $N$ are removed. Here a particle is said to be of generation $j$ if it was generated by branching of a particle of 
generation $j-1$. By the strong Markov property applied $N$ times (i.e. conditioning successively with respect to the first branching event), we have

$$
E\left(\int_{0}^{\infty} \mathrm{d} t \phi_{t}^{(0, v) ; N}(A)\right)=\left(\sum_{n=0}^{N-1}\left(\left[{ }^{\kappa} U \kappa\right] \rho\right)^{n}\right){ }^{\kappa} U(v, A)
$$

with all notations as in 2.1, and monotone convergence as $N \rightarrow \infty$, along with the proof of Lemma 1.4, concludes the proof.

2.3. Proposition. Under 1.1 and 1.3 , subprocesses $\phi:=\phi^{(0, v)}$, with $v \in \mathbb{R}^{d}$ arbitrary, have

(a) a.s. only finitely many jumps in finite time intervals, thus in particular $\zeta^{\phi}=+\infty$ a.s.;

(b) finite expected first return time to $\Delta: E\left(R^{\phi}\right)<\infty$.

Proof. By 2.2 together with Assumption 1.3 and Lemma 1.4, we have for $\phi:=\phi^{(0, v)}$

$$
E\left(R^{\phi} \wedge \zeta^{\phi}\right) \leqslant E\left(\int_{0}^{R^{\phi} \wedge \zeta^{\phi}} \mathrm{d} s \phi_{s}\left(\mathbb{R}^{d}\right)\right)={ }^{\gamma} R\left(v, \mathbb{R}^{d}\right)<\infty .
$$

In particular, trajectories of the process

$$
A_{t}^{\phi}:=\int_{0}^{t} \mathrm{~d} s \phi_{s}\left(\mathbb{R}^{d}\right)=\int_{0}^{R^{\phi} \wedge \zeta^{\phi} \wedge t} \mathrm{~d} s \phi_{s}\left(\mathbb{R}^{d}\right), \quad t \geqslant 0,
$$

are continuous on $\left[0, \infty\left[\right.\right.$, strictly increasing before time $R^{\phi} \wedge \zeta^{\phi}$, and have $\left(A^{\phi}\right)_{R^{\phi} \wedge \zeta^{\phi}}<\infty$ a.s. By 1.1 the branching rate $\kappa(\cdot)$ is continuous and bounded on $\mathbb{R}^{d}$. Hence all trajectories of

$$
B_{t}^{\phi}:=\int_{0}^{t} \mathrm{~d} s \phi_{s}(\kappa)=\int_{0}^{R^{\phi} \wedge \zeta^{\phi} \wedge t} \mathrm{~d} s \phi_{S}(\kappa), \quad t \geqslant 0,
$$

are continuous on $\left[0, \infty\right.$ [, strictly increasing before time $R^{\phi} \wedge \zeta^{\phi}$, and have $\left(B^{\phi}\right)_{R^{\phi} \wedge \zeta^{\phi}}<\infty$ a.s. But $B^{\phi}$ is the compensator of the process $N^{\phi}=\left(N_{t}^{\phi}\right)_{t}$ counting jump events in $\phi$ up to time $t$. So the path properties of $B^{\phi}$ imply that a.s. $N^{\phi}$ can have at most finitely many jumps over finite time intervals. So there is no accumulation of jumps in $\phi$, i.e. $\zeta^{\phi}=\infty$ a.s.

Thus we have proved that $E\left(R^{\phi}\right)<\infty$, where $R^{\phi}$ is the first passage to $\Delta$ which occurs after at most finitely many jumps in the trajectory of $\phi$.

2.4. Proposition. Assume 1.1 and 1.3. For immigration laws $\pi \in \mathcal{M}^{1}\left(\mathbb{R}^{d}\right)$ satisfying (8), the branching diffusion with immigration $\eta$ has the property $\mathrm{P} 1$.

Proof. Decompose the process $\eta$ into subprocesses $\phi^{\left(0, x^{i}\right)}, 1 \leqslant i \leqslant l$, where $x=\left(x^{1}, \ldots, x^{l}\right)$ is the initial configuration, and $\phi^{\left(T_{j}^{I}, \zeta_{j}^{I}\right)}$ stemming from the $j$-th immigration, $j \geqslant 1$.

Proposition 2.3 applied to every $\phi^{\left(0, x^{i}\right)}$ shows that the descendance of the initial configuration $x=\left(x^{1}, \ldots, x^{l}\right)$ will be extinct in finite time a.s., without accumulation of jumps in finite time intervals. 
Proposition 2.3 plus condition (8)

$$
\pi^{\gamma} R(A)=E_{\pi}\left(\int_{0}^{\infty} \mathrm{d} t 1_{A}\left(\xi_{t}\right) \mathrm{e}^{-\int_{0}^{t} \mathrm{~d} s[\kappa(1-\rho)]\left(\xi_{s}\right)}\right)=E\left(\int_{0}^{\infty} \mathrm{d} t \phi_{t}^{\left(T_{j}^{I}, \zeta_{j}^{I}\right)}(A)\right), \quad A \in \mathcal{B}\left(\mathbb{R}^{d}\right),
$$

shows that the same assertion holds for every subprocess $\phi^{\left(T_{j}^{I}, \zeta_{j}^{I}\right)}$.

The point process $\left(T_{j}^{I}, \zeta_{j}^{I}\right)_{j \geqslant 1}$ is a Poisson point process with intensity $c \mathrm{~d} s \pi(\mathrm{d} v)$ on $(0, \infty) \times \mathbb{R}^{d}$. Hence for immigration laws $\pi$ satisfying (8), the branching diffusion $\eta$ has a.s. no accumulation of jumps in finite time intervals.

2.5. Proposition. Assume 1.1 and 1.3. For immigration laws $\pi \in \mathcal{M}^{1}\left(\mathbb{R}^{d}\right)$ satisfying (8), the branching diffusion with immigration $\eta$ has the property $\mathrm{P} 2$.

Proof. (1) We know from Proposition 2.3 that under 1.1 and 1.3 the descendance of particles belonging to an arbitrary initial configuration $x \in S$ will be extinct a.s. in finite time.

We shall prove that $E_{\Delta}\left(R^{\eta}\right)<\infty$, where $R^{\eta}=R$ is the time of first return of a branching diffusion with immigration $\eta$ starting from $\eta_{0}=\Delta$, the void configuration, to $\Delta$.

Once this is proved, the measure $m$ defined on $S$ by (3) is necessarily a finite measure on $S$, and subsets $F \in \mathcal{B}(S)$ with $m(F)>0$ will be visited infinitely often by the process $\eta$, under arbitrary choice of a starting configuration $x \in S$. Hence $\eta$ is recurrent in the sense of Harris; in view of the ratio limit theorem, $m$ is the invariant measure of $\eta$, unique up to constant multiples, and $\eta$ is recurrent positive. So property P2 will be a consequence of $E_{\Delta}\left(R^{\eta}\right)<\infty$.

In fact, it is sufficient to prove

$$
E_{\pi}\left(R^{\eta}\right)<\infty
$$

since $\mathcal{L}\left(\eta_{T_{1}} \mid \eta_{0}=\Delta\right)$ is the immigration law $\pi$ viewed as a law on $S$, and since $E_{\Delta}\left(T_{1}\right)=\frac{1}{c}$.

Our proof of (24) is similar to an argument given by Zubkov [19, Proof of Theorem 1'] for classical branching processes with immigration. In the proof of (24), we assume throughout that $\eta$ starts at time $t=0$ from the initial law $\pi$.

(2) Distinguishing whether or not descendants of the initial population $\eta_{0}$ are still alive at time $t$, we write

$$
P_{\pi}\left(R^{\eta}>t\right)=P_{\pi}\left(R^{\phi}>t\right)+P_{\pi}\left(R^{\phi} \leqslant t, R^{\eta}>t\right)=P_{\pi}\left(R^{\phi}>t\right)+P_{\pi}\left(T_{1}^{I}<R^{\phi} \leqslant t, R^{\left(\eta^{\left(T_{1}^{I}, \zeta_{1}^{I}\right)}\right)}>t\right)
$$

because of

$$
\eta_{t}=\phi_{t}+\sum_{j=1}^{\infty} 1_{\llbracket T_{j}^{I}, \infty \llbracket}(t) \phi_{t}^{\left(T_{j}^{I}, \zeta_{j}^{I}\right)}=\phi_{t}+\eta_{t}^{\left(T_{1}^{I}, \zeta_{1}^{I}\right)}
$$

Conditioning with respect to $\mathcal{F}_{T_{1}^{I}}$ we get

$$
\begin{aligned}
P_{\pi}\left(R^{\eta}>t\right) & =P_{\pi}\left(R^{\phi}>t\right)+\int_{0}^{t} \mathrm{~d} s c \mathrm{e}^{-c s} P_{\pi}\left(s<R^{\phi} \leqslant t\right) P_{\pi}\left(R^{\eta}>t-s\right) \\
& \leqslant P_{\pi}\left(R^{\phi}>t\right)+\int_{0}^{t} \mathrm{~d} s c \mathrm{e}^{-c s} P_{\pi}\left(R^{\phi}>s\right) P_{\pi}\left(R^{\eta}>t-s\right) .
\end{aligned}
$$

Write $H(\mathrm{~d} s)$ for the finite measure on $(0, \infty)$

$$
H(\mathrm{~d} s):=\mathrm{d} s c \mathrm{e}^{-c s} P_{\pi}\left(R^{\phi}>s\right)
$$


with total mass $H((0, \infty))<1$ (compare with the exponential law with parameter $c$ ) and finite first moment $\int_{0}^{\infty} s H(\mathrm{~d} s)<\infty$. Then the desired recursion is

$$
P_{\pi}\left(R^{\eta}>t\right) \leqslant P_{\pi}\left(R^{\phi}>t\right)+\int_{0}^{t} H(\mathrm{~d} s) P_{\pi}\left(R^{\eta}>t-s\right) .
$$

If $t$ tends to $\infty$ in (25), we get with dominated convergence and 2.3(b)

$$
P_{\pi}\left(R^{\eta}=+\infty\right) \leqslant H((0, \infty)) P_{\pi}\left(R^{\eta}=+\infty\right)
$$

and thus

$$
R^{\eta}<\infty \quad P_{\pi} \text {-a.s. }
$$

(3) Now we can prove that $E_{\pi}\left(R^{\eta}\right)$ is indeed finite. By Proposition 2.3(b) and (26),

$$
u:=\mathcal{L}_{\pi}\left(R^{\eta}\right), \quad v:=\mathcal{L}_{\pi}\left(R^{\phi}\right), \quad h:=\frac{H}{H((0, \infty))}
$$

are probability distributions on $(0, \infty)$ with Laplace transforms $\psi_{u}, \psi_{v}, \psi_{h}$ such that as $\lambda \downarrow 0$

$$
\begin{aligned}
& \frac{1-\psi_{v}(\lambda)}{\lambda}=\int_{0}^{\infty} \mathrm{d} t \mathrm{e}^{-\lambda t} P_{\pi}\left(R^{\phi}>t\right) \uparrow E_{\pi}\left(R^{\phi}\right)<\infty, \\
& \frac{1-\psi_{h}(\lambda)}{\lambda} \uparrow \int_{0}^{\infty} \operatorname{sh}(\mathrm{d} s)<\infty
\end{aligned}
$$

whereas

$$
\psi_{u}(\lambda) \uparrow 1 \quad \text { as } \lambda \downarrow 0 .
$$

The inequality (25) now gives

$$
\frac{1-\psi_{u}(\lambda)}{\lambda} \leqslant \frac{1-\psi_{v}(\lambda)}{\lambda}+H((0, \infty)) \frac{1-\psi_{u * h}(\lambda)}{\lambda}
$$

where $\psi_{u * h}(\lambda)=\psi_{u}(\lambda) \psi_{h}(\lambda)=\psi_{u}(\lambda)\left(1-\left(1-\psi_{h}(\lambda)\right)\right)$, and thus

$$
\lim _{\lambda \downarrow 0}\left((1-H((0, \infty))) \frac{1-\psi_{u}(\lambda)}{\lambda}\right) \leqslant E_{\pi}\left(R^{\phi}\right)+\int_{0}^{\infty} s H(\mathrm{~d} s)<\infty .
$$

Thus we have shown that

$$
E_{\pi}\left(R^{\eta}\right)=\lim _{\lambda \downarrow 0} \frac{1-\psi_{u}(\lambda)}{\lambda}<\infty
$$

which is (24), and the proof of Proposition 2.5 is complete.

2.6. Proposition. Assume 1.1 and 1.3. For immigration laws $\pi \in \mathcal{M}^{1}\left(\mathbb{R}^{d}\right)$ satisfying (8), the branching diffusion with immigration $\eta$ has the property $\mathrm{P} 3$, and

$$
\bar{m}(A)=C \pi^{\gamma} R(A)=C E_{\pi}\left(\int_{0}^{\infty} \mathrm{d} t 1_{A}\left(\xi_{t}\right) \mathrm{e}^{-\int_{0}^{t} \mathrm{~d} s[\kappa(1-\rho)]\left(\xi_{s}\right)}\right)
$$

with $C=c E_{\Delta}(R)$. 
Proof. Under 1.1 and 1.3, fix $\pi \in \mathcal{M}^{1}\left(\mathbb{R}^{d}\right)$ such that (8) holds. By Proposition 2.5, the process $\eta$ is ergodic with invariant measure $m$ given by (3), with total mass $m(S)=E_{\Delta}(R)$. Let $R=R^{1}, R^{2}, \ldots$ denote successive passage times of $\eta$ to $\Delta$. Let $\eta$ start from $\eta_{0}=\Delta$ and consider the increasing process

$$
A_{t}:=\int_{0}^{t} \mathrm{~d} s \eta_{s}\left(\mathbb{R}^{d}\right), \quad t \geqslant 0 .
$$

By ergodicity, the ratio limit theorem for the additive functional $A$ gives

$$
\frac{A_{t}}{t} \longrightarrow \frac{\int_{S} m(\mathrm{~d} x) l(x)}{\int_{S} m(\mathrm{~d} x) 1} \leqslant \infty
$$

a.s. as $t \rightarrow \infty$. Compare $A$ to the process (which is not an additive functional of $\eta$ )

$$
B_{t}:=\sum_{j=1}^{\infty} 1_{\left\{T_{j}^{I}<t\right\}} \int_{T_{j}^{I}}^{D_{j}^{I}} \mathrm{~d} s \phi_{s}^{\left(T_{j}^{I}, \zeta_{j}^{I}\right)}\left(\mathbb{R}^{d}\right), \quad D_{j}^{I}:=R^{\left(\phi^{\left(T_{j}^{I}, \zeta_{j}^{I}\right)}\right)}
$$

where $D_{j}^{I}$ is the death time of $\phi^{\left(T_{j}^{I}, \zeta_{j}^{I}\right)}$. The variables $\int_{T_{j}^{I}}^{D_{j}^{I}} \mathrm{~d} s \phi_{s}^{\left(T_{j}^{I}, \zeta_{j}^{I}\right)}$ are i.i.d. and independent of the point process of immigration times. This point process of immigration times is Poisson with intensity $c$, so 2.2 and (8) together with a classical law of large numbers gives

$$
\frac{B_{t}}{t} \longrightarrow c\left[\pi^{\gamma} R\right]\left(\mathbb{R}^{d}\right)<\infty
$$

a.s. as $t \rightarrow \infty$. Since $A_{R^{j}}=B_{R^{j}}$ for all $j$ where the sequence $R^{j}$ is increasing to $\infty$, comparison of (27) and (28) shows

$$
\bar{m}\left(\mathbb{R}^{d}\right)=\int_{S} m(\mathrm{~d} x) l(x)=c E_{\Delta}(R)\left[\pi^{\gamma} R\right]\left(\mathbb{R}^{d}\right)<\infty
$$

which is the property P3. Now repeating the same argument with a set $A \in \mathcal{B}\left(\mathbb{R}^{d}\right)$ instead of $A=\mathbb{R}^{d}$ identifies the measure $\bar{m}$ as $C \pi^{\gamma} R$.

2.7. Proposition. Under 1.1, assume that the branching diffusion $\eta$ has properties $\mathrm{P} 1+\mathrm{P} 2+\mathrm{P} 3$ for arbitrary choice of an immigration law $\pi \in \mathcal{M}^{1}\left(\mathbb{R}^{d}\right)$.

Then $v \rightarrow{ }^{\gamma} R\left(v, \mathbb{R}^{d}\right)$ is a bounded function.

Proof. (1) Repeating the argument in the proof of 2.6, we see that under P1 + P2 + P3, the invariant occupation measure $\bar{m}$ is necessarily of form $C \pi^{\gamma} R$ if $\pi$ is the immigration law.

(2) Assuming only 1.1, consider the function

$$
v \rightarrow V(v):={ }^{\gamma} R\left(v, \mathbb{R}^{d}\right)=E_{v}\left(\int_{0}^{\infty} \mathrm{d} t \mathrm{e}^{-\int_{0}^{t} \mathrm{~d} s[\kappa(1-\rho)]\left(\xi_{s}\right)}\right) \in[0, \infty] .
$$

If $\bar{m}\left(\mathbb{R}^{d}\right)=C \pi(V)$ is finite for arbitrary $\pi \in \mathcal{M}^{1}\left(\mathbb{R}^{d}\right)$, then $V(\cdot)$ is necessarily finite-valued (consider $\pi:=\delta_{v}$, $\left.v \in \mathbb{R}^{d}\right)$. Also $V(\cdot)$ is necessarily bounded: if not, we could select a sequence of points $\left(v_{n}\right)_{n}$ in $\mathbb{R}^{d}$ such that $n \leqslant V\left(v_{n}\right)<n+1$, and could consider a law $\pi$ of type $\operatorname{cst} \sum_{n} \frac{1}{n^{2}} \delta_{v_{n}}$ to obtain a contradiction.

2.8. Proof of Theorem 1.6. Theorem 1.6 is proved by Propositions $2.4-2.7$ together. 
2.9. Proof of Theorem 1.7. We assume 1.1 and 1.3, take $\sigma(\cdot)$ bounded, and fix an immigration law $\pi$ such that (8) holds. We shall show that the invariant occupation measure $\bar{m}$ - explicitly known by Theorem 1.6 - satisfies

$$
\text { Af } \in L^{1}(\bar{m}), \quad \bar{m}(A f-\gamma f)=-C \pi(f), \quad \text { for all } f \in \mathcal{C}_{b}^{2}\left(\mathbb{R}^{d}, \mathbb{R}\right)
$$

where $A$ is the Markov generator of the diffusion $\xi$

$$
A f(v)=\frac{1}{2} \sum_{i, j=1}^{d} a_{i, j}(v) \frac{\partial^{2} f}{\partial v_{i} \partial v_{j}}(v)+\sum_{i=1}^{d} b_{i}(v) \frac{\partial f}{\partial v_{i}}(v) .
$$

1. For functions $f \in \mathcal{C}_{b}:=\mathcal{C}_{b}\left(\mathbb{R}^{d}, \mathbb{R}\right)$ and for $x \in S$ write $\bar{f}(x):=x(f)$, i.e.

$$
\bar{f}(x)=\sum_{i=1}^{l} f\left(x^{i}\right) \quad \text { if } x=\left(x^{1}, \ldots, x^{l}\right) \text { with } l \geqslant 1 \text {, and } \bar{f}(\Delta)=0 .
$$

Under our assumptions, the invariant occupation measure $\bar{m}$ is explicitly known by theorem 1.6 and is a finite measure, so we have $m(\bar{f})=\bar{m}(f)<\infty$ for all $f \in \mathcal{C}_{b}$.

2. Consider $f \in \mathcal{C}^{2}$. The Ito (or Dynkin) formula for $\eta$ - obtained from Ito's formula for $\left(\bar{f}\left(\eta_{t}\right)\right)_{t}$ between successive jump times, and compensating the jumps - is

$$
\bar{f}\left(\eta_{t}\right)-\bar{f}\left(\eta_{0}\right)=\int_{0}^{t} L \bar{f}\left(\eta_{s}\right) \mathrm{d} s+N_{t}^{c}+N_{t}^{d}, \quad f \in \mathcal{C}^{2},
$$

where $L$ is the infinitesimal generator of $\eta$

$$
\begin{aligned}
L \bar{f}(x) & =\sum_{i=1}^{l(x)}\left(A f\left(x^{i}\right)-[\kappa(1-\rho)]\left(x^{i}\right) f\left(x^{i}\right)\right)+c \pi(f) \\
& =\overline{(A f-\gamma f)}(x)+c \pi(f),
\end{aligned}
$$

where $N^{c}$ is a continuous locally square integrable local martingale with angle bracket

$$
\left\langle N^{c}\right\rangle_{t}=\int_{0}^{t} \overline{\nabla^{\top} f \cdot a \cdot \nabla f}\left(\eta_{s}\right) \mathrm{d} s,
$$

and where $N^{d}$ is the purely discontinuous local martingale

$$
N_{t}^{d}=\sum_{n=1}^{\infty} 1_{\left\{T_{n} \leqslant t\right\}}\left(\bar{f}\left(\eta_{T_{n}}\right)-\bar{f}\left(\eta_{T_{n}^{-}}\right)\right)-\left(c \pi(f) t+\int_{0}^{t} \overline{[\kappa(\rho-1) f]}\left(\eta_{s}\right) \mathrm{d} s\right) .
$$

3. Consider the branching diffusion $\eta$ with immigration law $\pi$ as a stationary process, i.e. take $\mathcal{L}\left(\eta_{0}\right)=\frac{1}{E_{\Delta}(R)} m$. Consider functions $f \in \mathcal{C}_{b}^{2}$.

Since $[\kappa(1-\rho)]$ and $\sigma$ are bounded and since $\bar{m}(g)<\infty$ for bounded $g$, the local martingales $N^{c}$ and $N^{d}$ of step 2 are now martingales.

Then (30) for $f \in \mathcal{C}_{b}^{2}$ shows

$$
L \bar{f} \in L^{1}(m), \quad 0=m(L \bar{f}), \quad \text { for all } f \in \mathcal{C}_{b}^{2}
$$

which gives

$$
\text { Af } \in L^{1}(\bar{m}), \quad \bar{m}(A f-\gamma f)=-C \pi(f), \quad \text { for all } f \in \mathcal{C}_{b}^{2}
$$

with $C=c E_{\Delta}\left(R^{\eta}\right)$. We have proved that the invariant occupation measure $\bar{m}$ solves (10). 


\section{Invariant occupation density}

This section deals with existence and properties of a density for the invariant occupation measure of Section 1. The results stated in Subsections 3.1 and 3.2 will be proved in Section 4.

\subsection{Probabilistic approach}

We will need resolvents for the diffusion $\xi$ of (1) and for an associated diffusion $\xi \leftarrow$ introduced in (31) below in case where $a=\sigma \sigma^{\top}$ is spatially constant, $\xi^{\leftarrow}$ is the process $\xi$ run backwards in time - with appropriate 'killing rates'. We will also need solutions of SDE's (1) and (31) in form of a stochastic flow of $\mathcal{C}^{2}$-diffeomorphisms, see Kunita [11, Corollary 4.6.5].

We have to strengthen the Assumptions 1.1 and 1.3 used in Section 1.

3.1. Assumption. We have 1.1 together with

$$
\sigma \in \mathcal{C}_{b}^{4}\left(\mathbb{R}^{d}, \mathbb{R}^{d \times m}\right), \quad b \in \mathcal{C}_{(b)}^{3}\left(\mathbb{R}^{d}, \mathbb{R}^{d}\right), \quad \gamma \in \mathcal{C}_{b}^{2}\left(\mathbb{R}^{d}, \mathbb{R}\right) .
$$

3.2. Assumption. The function in (6)

$$
v \longrightarrow \gamma_{R}\left(v, \mathbb{R}^{d}\right)=E_{v}\left(\int_{0}^{\infty} \mathrm{d} t \mathrm{e}^{-\int_{0}^{t} \mathrm{~d} s \gamma\left(\xi_{s}\right)}\right)
$$

is bounded on $\mathbb{R}^{d}$.

Hence by Theorem 1.6, the branching diffusion with immigration $\eta$ has properties $\mathrm{P} 1+\mathrm{P} 2+\mathrm{P} 3$ for arbitrary choice of an immigration law $\pi \in \mathcal{M}^{1}\left(\mathbb{R}^{d}\right)$.

Under 3.1, we introduce a diffusion $\xi^{\leftarrow}$ associated to (1), with drift $b^{\leftarrow} \in \mathcal{C}_{(b)}^{3}$ :

$$
\mathrm{d} \xi_{t}^{\leftarrow}=b^{\leftarrow}\left(\xi_{t}^{\leftarrow}\right) \mathrm{d} t+\sigma\left(\xi_{t}^{\leftarrow}\right) \mathrm{d} \widetilde{W}_{t}, \quad b_{i}^{\leftarrow}(v):=-b_{i}(v)+\sum_{k=1}^{d} \frac{\partial a_{i, k}}{\partial v_{k}}(v), \quad 1 \leqslant i \leqslant d .
$$

We define a 'killing rate' $\gamma^{\leftarrow} \in \mathcal{C}_{b}^{2}$ (this is abuse of language; $\gamma^{\leftarrow}$ may take negative values)

$$
\gamma^{\leftarrow}(v):=\gamma(v)+\sum_{i=1}^{d} \frac{\partial b_{i}}{\partial v_{i}}(v)-\frac{1}{2} \sum_{i, k=1}^{d} \frac{\partial^{2} a_{i, k}}{\partial v_{i} \partial v_{k}}(v)
$$

for $\xi^{\leftarrow}$ in order to consider the resolvent $\gamma^{\leftarrow} R^{\leftarrow}$ : for $f \geqslant 0$ measurable and $v \in \mathbb{R}^{d}$

$$
\left(\gamma^{\leftarrow} R^{\leftarrow} f\right)(v)=E_{v}\left(\int_{0}^{\infty} \mathrm{d} t f\left(\xi_{t}^{\leftarrow}\right) \mathrm{e}^{-\int_{0}^{t} \mathrm{~d} s \gamma^{\leftarrow}\left(\xi_{s}^{\leftarrow}\right)}\right) \in[0, \infty]
$$

Note that $\gamma^{\leftarrow} R^{\leftarrow}$ is the adjoint resolvent to ${ }^{\gamma} R$.

3.3. Remark. (a) Write $\xi \leftarrow \leftarrow$ for the diffusion $\xi$ run backwards in time: then $\xi \leftarrow \leftarrow$ has drift $b \leftarrow \leftarrow$ with components

$$
b_{i}^{\leftarrow \leftarrow}(v):=-b_{i}(v)+\sum_{l=1}^{m} \sum_{k=1}^{d} \sigma_{k, l}(v) \frac{\partial \sigma_{i, l}}{\partial v_{k}}(v), \quad 1 \leqslant i \leqslant d,
$$


see e.g. Kunita [11, pp. 131-135]. This is seen from the Stratonovich form of SDE (1) where the Stratonovich integral is symmetric with respect to passage to the backwards diffusion, see Ikeda and Watanabe [9, pp. 100-101].

In the special case of spatially constant $\sigma, b^{\leftarrow \leftarrow}$ and $b^{\leftarrow}$ coincide.

(b) Under 3.1 and 3.2, the resolvent kernel (33) cannot yet be expected to be a finite kernel. As an example, let $\xi$ in (1) be an ergodic Ornstein-Uhlenbeck process $\mathrm{d} \xi_{t}=-\vartheta \xi_{t} \mathrm{~d} t+\mathrm{d} W_{t}, \vartheta>0$, with spatially constant killing rate $\gamma>0$. Then $\xi \leftarrow$ in (31) is a transient Ornstein-Uhlenbeck process, and $\gamma^{\leftarrow}=\gamma-\vartheta$ defined in (32) is negative for $\vartheta>\gamma$.

3.4. Assumption. The function

$$
v \longrightarrow \gamma^{\leftarrow} R^{\leftarrow}\left(v, \mathbb{R}^{d}\right)=E_{v}\left(\int_{0}^{\infty} \mathrm{d} t \mathrm{e}^{-\int_{0}^{t} \mathrm{~d} s \gamma^{\leftarrow}\left(\xi_{s}^{\leftarrow}\right)}\right)
$$

is bounded on $\mathbb{R}^{d}$.

\subsection{Theorem. Assume 3.1, 3.2, 3.4, and define}

$$
\mathcal{G}:=\left\{g \in \mathcal{C}_{b}^{2}: g \in L^{1}(\lambda) \text { and } \gamma^{\leftarrow} R^{\leftarrow} g \in L^{1}(\lambda)\right\} .
$$

If the branching diffusion with immigration $\eta$ has immigration law $\pi \in \mathcal{M}^{1}\left(\mathbb{R}^{d}\right)$ satisfying

$$
\pi(\mathrm{d} v):=g(v) \mathrm{d} v \text { where } g \text { belongs to class } \mathcal{G},
$$

then the invariant occupation measure $\bar{m}=C \pi^{\gamma} R$ in 1.6 is Lebesgue absolutely continuous. The density is

$$
\frac{\mathrm{d} \bar{m}}{\mathrm{~d} \lambda}=C^{\gamma \leftarrow} R^{\leftarrow} g
$$

which is bounded on $\mathbb{R}^{d}$.

There are two simple conditions leading to continuous Lebesgue densities $\frac{\mathrm{d} \bar{m}}{\mathrm{~d} \lambda}$ on $\mathbb{R}^{d}$ :

3.6. Lemma. Assume 3.1, 3.2, 3.4.

(a) If 3.2 is strengthened to

$$
\inf _{v \in \mathbb{R}^{d}} \gamma(v)>0
$$

then

$$
\text { g nonnegative and in } \mathcal{C}_{b}^{2} \cap L^{1}(\lambda) \text { implies }{ }^{\leftarrow} R^{\leftarrow} g \in L^{1}(\lambda) \text {. }
$$

(b) If 3.4 is strengthened to

$$
\inf _{v \in \mathbb{R}^{d}} \gamma^{\leftarrow}(v)>0
$$

then

$$
g \in \mathcal{C}_{b}^{2} \text { implies } \gamma^{\leftarrow} R^{\leftarrow} g \in \mathcal{C}_{b}^{0} .
$$

The following example illustrates why in $3.5+3.6$ - under the conditions made so far - we obtain not more than continuity for the invariant occupation density $\frac{\mathrm{d} \bar{m}}{\mathrm{~d} \lambda}$. In a completely 'smooth' framework with stronger conditions, better results (see Subsection 3.2) are available. 
3.7. Example. We continue the one-dimensional example of 3.3(b): Consider an Ornstein-Uhlenbeck process $\xi=\left(\xi_{t}\right)_{t} \geqslant 0$

$$
\mathrm{d} \xi_{t}=-\vartheta \xi_{t} \mathrm{~d} t+\mathrm{d} W_{t}
$$

with parameter $\vartheta \in \mathbb{R}$, and fix some spatially constant killing rate $\gamma>0$.

Then $\xi^{\leftarrow}$ of (31) is Ornstein-Uhlenbeck of parameter $-\vartheta$, which is as in 3.3(a) the diffusion $\xi$ running backwards in time; the stochastic flow corresponding to $\xi^{\leftarrow}$ is

$$
\psi \leftarrow(t, v)=\mathrm{e}^{\vartheta t} v+\mathrm{e}^{\vartheta t} \int_{0}^{t} \mathrm{e}^{-\vartheta s} \mathrm{~d} \widetilde{W}_{s}, \quad t \geqslant 0, v \in \mathbb{R},
$$

where $\frac{\partial}{\partial v} \psi^{\leftarrow}(t, v)=\mathrm{e}^{\vartheta t}$ is independent of $v$, and $\frac{\partial^{2}}{\partial v^{2}} \psi^{\leftarrow}(t, v)=0$.

The resolvent defined in $(32)+(33)$ is

$$
\gamma^{\leftarrow} R^{\leftarrow} g(v)=E\left(\int_{0}^{\infty} \mathrm{d} t g\left(\psi^{\leftarrow}(t, v)\right) \mathrm{e}^{-\gamma^{\leftarrow} t}\right), \quad \gamma^{\leftarrow}=\gamma-\vartheta .
$$

All assumptions of $3.5+3.6$ are satisfied provided $\gamma>\vartheta$; in this case, with choice $g=\frac{\mathrm{d} \pi}{\mathrm{d} \lambda} \in \mathcal{C}_{b}^{2}$, we have from $3.5+3.6$ a continuous invariant occupation density $\frac{\mathrm{d} \bar{m}}{\mathrm{~d} \lambda}$ given by (35) multiplied with the constant $C$.

(i) Consider the case $\vartheta \leqslant 0$ where $\xi \leftarrow$ is recurrent (positive or null).

Since $\gamma-(k+1) \vartheta$ is strictly positive for all $k \in \mathbb{N}_{0}$, resolvents (35) with arbitrary $g \in \mathcal{C}_{b}^{m}$ belong to $\mathcal{C}_{b}^{m}$; the $k$-th derivatives are

$$
\left(\gamma^{\leftarrow} R^{\leftarrow} g\right)^{(k)}(v)=E\left(\int_{0}^{\infty} \mathrm{d} t g^{(k)}\left(\psi^{\leftarrow}(t, v)\right) \mathrm{e}^{-[\gamma-(k+1) \vartheta] t}\right), \quad 1 \leqslant k \leqslant m .
$$

(ii) Consider the case $\vartheta \in(0, \gamma)$ where $\xi \leftarrow$ is transient.

For given $k, \gamma-(k+1) \vartheta$ in (36) is positive only for $\vartheta$ sufficiently small, so resolvents (35) with arbitrary $g \in \mathcal{C}_{b}^{m}$ will in general only have the property

$$
\gamma^{\leftarrow} R^{\leftarrow} g \in \mathcal{C}_{b}^{m \wedge k} \quad \text { on the interval } \vartheta \in\left[\frac{\gamma}{k+2}, \frac{\gamma}{k+1}\right), \quad k \in \mathbb{N}_{0}
$$

In particular, for $\frac{\gamma}{2} \leqslant \vartheta<\gamma$, this is continuity of $\gamma^{\leftarrow} R^{\leftarrow} g$ as stated in 3.6(b).

(iii) In case $\vartheta \geqslant \gamma$, Assumption 3.4 is violated.

\subsection{Analytic approach}

A smooth Lebesgue density can be obtained for the invariant occupation measure $\bar{m}=C \pi^{\gamma} R$ in Theorem 1.6 if we assume bounded smooth coefficients

$$
\sigma(\cdot) \in \mathcal{C}_{b}^{\infty}\left(\mathbb{R}^{d}, \mathbb{R}^{d \times m}\right), \quad b(\cdot) \in \mathcal{C}_{b}^{\infty}\left(\mathbb{R}^{d}, \mathbb{R}^{d}\right), \quad \gamma(\cdot) \in \mathcal{C}_{b}^{\infty}\left(\mathbb{R}^{d}, \mathbb{R}\right)
$$

together with uniform ellipticity

$$
\inf _{v \in \mathbb{R}^{d}} \inf _{\beta \in \mathbb{R}^{d},|\beta|=1} \beta^{\top} a(v) \beta>0 \quad\left(a=\sigma \sigma^{\top}\right)
$$

for the one-particle motion (1) and the mass reduction rate $\gamma$. Note that the pair of conditions (37) $+(38)$ for $\xi$ and $\gamma$ implies the corresponding pair of conditions for the associated $\xi^{\leftarrow}$ and $\gamma^{\leftarrow}$ defined in (31) and (32), and vice versa. 
For the diffusion $\xi$ of (1), the uniform ellipticity condition (38) can be replaced by the uniform general Hörmander condition, introduced by Hörmander [7], see Williams [18], Kusuoka and Stroock [12] and Cattiaux [2]: there is some $N$ such that

$$
\inf _{v \in \mathbb{R}^{d}} \inf _{\beta \in \mathbb{R}^{d},|\beta|=1} \sum_{V \in \mathcal{V}_{N}(v)}\left(\beta^{\top} V\right)^{2}>0
$$

where the set $\mathcal{V}_{N}(v)$ consists of $V^{0}, V^{1}, \ldots, V^{m}$ together with all Lie brackets of $V^{0}, V^{1}, \ldots, V^{m}$ up to order $N$ where

$$
V^{j} \text { is the } j \text {-th column in } \sigma(v), \quad 1 \leqslant j \leqslant m,
$$

and where $V^{0}$ is the drift at $v$ of Eq. (1) rewritten in Stratonovich form:

$$
V_{i}^{0}=b_{i}(v)-\frac{1}{2} \sum_{l=1}^{m} \sum_{j=1}^{d} \sigma_{j, l}(v) \frac{\partial \sigma_{i, l}}{\partial v_{j}}(v), \quad 1 \leqslant i \leqslant d .
$$

Here the Lie bracket $[V, \widetilde{V}]$ has components

$$
[V, \widetilde{V}]_{i}=\sum_{j=1}^{d}\left(V_{j} \frac{\partial \widetilde{V}_{i}}{\partial v_{j}}-\widetilde{V}_{j} \frac{\partial V_{i}}{\partial v_{j}}\right), \quad 1 \leqslant i \leqslant d,
$$

and the order of an iterated Lie bracket is the maximal degree of partial derivatives appearing in it.

We need conditions which are symmetric in $\xi, \gamma$ and the associated $\xi^{\leftarrow}, \gamma^{\leftarrow}$. The uniform general Hörmander condition for $\xi^{\leftarrow}$ is the condition (39) with drift $b(\cdot)$ in (40) replaced by $b^{\leftarrow}(\cdot)$. So we strengthen Assumption 3.1:

3.8. Assumption. We have 1.1 together with the bounded smoothness condition (37), and the uniform general Hörmander condition holds for both $\xi$ and $\xi^{\leftarrow}$.

The following theorem is then a corollary to results given in Cattiaux [2, Proposition 2.19, Remarques 2.21]:

\subsection{Theorem. Assume 3.8, 3.2, 3.4.}

Consider an immigration law $\pi \in \mathcal{M}^{1}\left(\mathbb{R}^{d}\right)$ with $\mathcal{C}_{b}^{\infty}$-density $g=\frac{\mathrm{d} \pi}{\mathrm{d} \lambda}$. Then $g$ belongs to class $\mathcal{G}$ defined in (34), and the Lebesgue density of $\bar{m}=C \pi^{\gamma} R$ in 3.5 is smooth:

$$
\frac{\mathrm{d} \bar{m}}{\mathrm{~d} \lambda}=C^{\gamma^{\leftarrow}} R^{\leftarrow} g \in \mathcal{C}^{\infty} .
$$

If $g=\frac{\mathrm{d} \pi}{\mathrm{d} \lambda}$ is in $\mathcal{S}$, then $\bar{m}$ has finite moments of arbitrary order.

Here $\mathcal{S}$ is the Schwartz space of all functions in $h \in \mathcal{C}^{\infty}\left(\mathbb{R}^{d}, \mathbb{R}\right)$ such that for arbitrary $N$

$$
\sup _{v \in \mathbb{R}^{d},\left(i_{1}, \ldots, i_{p}\right) \in\{1, \ldots, d\}^{p}, p \leqslant N}(1+|v|)^{N}\left|\frac{\partial^{p}}{\partial_{v_{i_{1}}} \ldots \partial_{v_{i_{p}}}} h(v)\right|<\infty,
$$

see Edwards [4].

By Theorem 3.9, we have - under the strong set of Assumptions 3.8, in particular (37), on the diffusive motions $\xi$ and $\xi^{\leftarrow}$, and under weak assumptions on the branching mechanism, expressing only that 'killing rates' $\gamma$ and $\gamma^{\leftarrow}$ have to satisfy $3.2+3.4$ - very satisfactory information on the invariant occupation measure whenever the immigration density is in $\mathcal{C}_{b}^{\infty}$.

The result in the 'smooth' context here relies on a strong duality, see Theorem 4.5, (49) + (51), and Remark 4.7 below. For immigration densities in class $\mathcal{S}$, this duality would have allowed to deduce the desired smooth density 
$\frac{\mathrm{d} \bar{m}}{\mathrm{~d} \lambda}=C^{\gamma} R^{\leftarrow} \frac{\mathrm{d} \pi}{\mathrm{d} \lambda}$ for the invariant occupation measure directly from Theorem 1.6. This duality is not available in the $\mathcal{C}^{2}$-context of Theorem 3.5 and Lemma 3.6.

We note that from a modelling point of view, or from the point of view of statistical inference, the strong assumptions needed for Theorem 3.9 - like (37) contained in 3.8 - are problematic; they rule out some simple and natural models like the Ornstein-Uhlenbeck Example 3.7.

\section{Proofs for Section 3}

We start with a careful look at the class of solutions to Eq. (10) under the stronger conditions of Theorem 3.5. We write for short ${ }^{\gamma} A$ for the mapping $f \rightarrow A f-\gamma f$ in (10).

4.1. Proposition. Assume 3.1 and 3.2. Then for arbitrary immigration law $\pi \in \mathcal{M}^{1}\left(\mathbb{R}^{d}\right)$, the invariant occupation measure $\bar{m}=C \pi^{\gamma} R$ is the unique solution to Eq. (10)

$$
\tilde{m}\left({ }^{\gamma} A f\right)=-C \pi(f), \quad f \in \mathcal{C}_{b}^{2},
$$

in the class of all finite measures $\tilde{m}$ on $\mathbb{R}^{d}$.

Proof. 1. Let $\left({ }^{\gamma} P_{t}\right)_{t \geqslant 0}$ denote the semigroup corresponding to $\xi$ and $\gamma$ :

$$
\gamma_{P_{t}}(v, f):=E_{v}\left(f\left(\xi_{t}\right) \mathrm{e}^{-\int_{0}^{t} \mathrm{~d} s \gamma\left(\xi_{s}\right)}\right), \quad v \in \mathbb{R}^{d}, f \text { nonnegative and measurable. }
$$

Since $\gamma=[\kappa(1-\rho)]$ is bounded, every ${ }^{\gamma} P_{t}(\cdot, \cdot)$ is a finite kernel on $\left(\mathbb{R}^{d}, \mathcal{B}\left(\mathbb{R}^{d}\right)\right)$. The resolvent kernel for the semigroup (41) appeared in Lemma 1.4.

2. 3.1 guarantees (a weaker variant of 3.1 would be enough here) that the diffusion $\xi$ of (1) can be constructed as a stochastic flow of $\mathcal{C}^{2}$-diffeomorphisms, see Kunita [11, Theorem 4.6.5, and pp. 72-73, 79-80, 85]. Write $(\psi(t, v))_{t \geqslant 0, v \in \mathbb{R}^{d}}$ for this flow. As in Kunita [10, pp. 210-223], there are $L^{p}$-bounds - uniformly in $v \in K, 0 \leqslant$ $t \leqslant T$ - for partial derivatives $\frac{\partial}{\partial v_{i}} \psi(t, v)$ or $\frac{\partial^{2}}{\partial v_{i} \partial v_{j}} \psi(t, v)$, for arbitrary $p>2$, and these bounds are of exponential type in $T$ (see also Cattiaux [2, Théorème (1.1)]). Using $\gamma \in \mathcal{C}_{b}^{2}$ by Assumption 3.1, and uniform integrability of spatial derivatives in (41), we get

$$
{ }^{\gamma} P_{t} f \in \mathcal{C}_{b}^{2} \text { for all } f \in \mathcal{C}_{b}^{2} \text { and all } 0 \leqslant t<\infty .
$$

(This argument cannot be extended to the resolvant ${ }^{\gamma} R f$, due to the exponential structure in $T$ of the bounds mentioned above.)

3. Under 3.1, we consider test functions $f \in \mathcal{C}_{\mathcal{K}}^{\infty}$. Then ${ }^{\gamma} A f \in \mathcal{C}_{\mathcal{K}}^{2}$, and from (41) and Ito formula

$$
\frac{\partial}{\partial t}{ }^{\gamma} P_{t} f(v)={ }^{\gamma} P_{t}(A f-\gamma f)(v)={ }^{\gamma} P_{t}{ }^{\gamma} A f(v)
$$

Hence ${ }^{\gamma} A$ is the infinitesimal generator of the semigroup (41), thus

$$
{ }^{\gamma} P_{t}{ }^{\gamma} A f={ }^{\gamma} A^{\gamma} P_{t} f, \quad f \in \mathcal{C}_{\mathcal{K}}^{\infty},
$$

and thus

$$
{ }^{\gamma} P_{T} f(v)-f(v)=\int_{0}^{T} \mathrm{~d} t^{\gamma} A^{\gamma} P_{t} f(v), \quad f \in \mathcal{C}_{\mathcal{K}}^{\infty} .
$$


For $f \in \mathcal{C}_{\mathcal{K}}^{\infty}$, the functions ${ }^{\gamma} P_{t} f$ and ${ }^{\gamma} A^{\gamma} P_{t} f$ are in $\mathcal{C}_{b}^{2}$, by (43). Hence we have for a finite measure $\tilde{m}$ satisfying (10)

$$
\tilde{m}(f)-\tilde{m}\left({ }^{\gamma} P_{T} f\right)=C \pi\left(\int_{0}^{T} \mathrm{~d} t{ }^{\gamma} P_{t} f\right), \quad f \in \mathcal{C}_{\mathcal{K}}^{\infty} .
$$

The resolvent ${ }^{\gamma} R f=\int_{0}^{\infty} \mathrm{d} t \gamma P_{t} f$ is a bounded function, by Assumption 3.2. Taking averages $\frac{1}{T^{\prime}} \int_{0}^{T^{\prime}} \mathrm{d} T \ldots$ in the last equation, we deduce as $T^{\prime} \rightarrow \infty$

$$
\tilde{m}=C \pi^{\gamma} R \text {. }
$$

This proves Proposition 4.1.

The key point for the results in Subsection 3.1 is the following lemma with formula (49) in its proof.

4.2. Lemma. Assume 3.1, 3.2 and 3.4. Then we have

$$
\left(\gamma^{\leftarrow} R^{\leftarrow} g,{ }^{\gamma} A f\right)=-(g, f), \quad g \in \mathcal{C}_{b}^{2} \cap L^{1}(\lambda), f \in \mathcal{C}_{b}^{2},
$$

with $(\cdot, \cdot)$ denoting the scalar product in $L^{2}(\lambda)$.

Proof. 1. Consider the semigroup associated to $(31)+(32)$

$$
\left(\gamma^{\leftarrow} P_{t}^{\leftarrow}\right)(v, f):=E_{v}\left(f\left(\xi_{t} \leftarrow\right) \mathrm{e}^{-\int_{0}^{t} \mathrm{~d} s \gamma^{\leftarrow}\left(\xi_{s}^{\leftarrow}\right)}\right), \quad v \in \mathbb{R}^{d}, f \geqslant 0 \text { measurable }
$$

In virtue of the strong Assumptions in 3.1, all assertions in steps 2, 3 of the proof of 4.1 concerning $\left({ }^{\gamma} P_{t}\right)_{t}$ have exact analogues in terms of $\left(\gamma^{\leftarrow} P_{t}^{\leftarrow}\right)_{t}$ : we have $b^{\leftarrow} \in \mathcal{C}_{(b)}^{3}, \sigma \in \mathcal{C}_{b}^{4}$, and have $\xi \leftarrow$ in form of a stochastic flow of $\mathcal{C}^{2}$-diffeomorphisms. We have $\gamma^{\leftarrow} \in \mathcal{C}_{b}^{2}$, so (46) is a finite kernel for $t$ fixed, and we have ${ }^{\leftarrow} P_{t}^{\leftarrow} f \in \mathcal{C}_{b}^{2}$ for $f \in \mathcal{C}_{b}^{2}$. As in the proof of 4.1, we see that the generator of the semigroup (46) is

$$
\gamma^{\leftarrow} A^{\leftarrow} f(v):=\frac{1}{2} \sum_{i, j=1}^{d} a_{i, j}(v) \frac{\partial^{2} f}{\partial v_{i} \partial v_{j}}(v)+\sum_{i=1}^{d} b_{i}^{\leftarrow}(v) \frac{\partial f}{\partial v_{i}}(v)-\gamma^{\leftarrow}(v) f(v) .
$$

2. Write $A^{*}$ for the adjoint of the Markov generator $A$ of $\xi$ in (29), see Williams [18] or Bass [1]:

$$
A^{*} f(v)=\frac{1}{2} \sum_{i, k=1}^{d} \frac{\partial^{2}\left(a_{i, k} f\right)}{\partial v_{i} \partial v_{k}}(v)-\sum_{i=1}^{d} \frac{\partial\left(b_{i} f\right)}{\partial v_{i}}(v), \quad f \in \mathcal{C}^{2}, v \in \mathbb{R}^{d} .
$$

Then we can combine $(31)+(32)+(48)$ to

$$
A^{*} f(v)=\frac{1}{2} \sum_{i, j=1}^{d} a_{i, j}(v) \frac{\partial^{2} f}{\partial v_{i} \partial v_{j}}(v)+\sum_{i=1}^{d} b_{i}^{\leftarrow}(v) \frac{\partial f}{\partial v_{i}}(v)-\left(\gamma^{\leftarrow}-\gamma\right)(v) f(v)
$$

which gives

$$
\gamma^{\leftarrow} A^{\leftarrow} f=\left(A^{*} f-\gamma f\right), \quad f \in \mathcal{C}^{2} .
$$

3. For every pair of functions $\tilde{g} \in \mathcal{C}^{2}, h \in \mathcal{C}_{\mathcal{K}}^{2}$ (thus we have ${ }^{\gamma} A h \in \mathcal{C}_{\mathcal{K}}^{0}$ ), we deduce from (49)

$$
\left(\gamma^{\leftarrow} A^{\leftarrow} \tilde{g}, h\right)=\left(A^{*} \tilde{g}, h\right)-(\gamma \tilde{g}, h)=(\tilde{g}, A h)-(\tilde{g}, \gamma h)=\left(\tilde{g},{ }^{\gamma} A h\right) .
$$

Starting from a function $g \in \mathcal{C}_{b}^{2}$, we have $\tilde{g}:=\gamma^{\leftarrow} P_{s}^{\leftarrow} g \in \mathcal{C}_{b}^{2}$ and thus 


$$
\left(\frac{\partial}{\partial s} \gamma^{\leftarrow} P_{s}^{\leftarrow} g, h\right)=\left(\gamma^{\leftarrow} A^{\leftarrow \gamma^{\leftarrow}} P_{s}^{\leftarrow} g, h\right)=\left(\gamma^{\leftarrow} P_{s}^{\leftarrow} g,{ }^{\gamma} A h\right)
$$

hence for $g \in \mathcal{C}_{b}^{2}, h \in \mathcal{C}_{\mathcal{K}}^{2}$, and $0<T<\infty$

$$
\int_{0}^{T} \mathrm{~d} s\left(\gamma^{\leftarrow} P_{s}^{\leftarrow} g,{ }^{\gamma} A h\right)=\left(\gamma^{\leftarrow} P_{T}^{\leftarrow} g, h\right)-(g, h)
$$

Now $\gamma^{\leftarrow} R^{\leftarrow}\left(\cdot, \mathbb{R}^{d}\right)=\int_{0}^{\infty} \mathrm{d} t \gamma^{\leftarrow} P_{t}^{\leftarrow}\left(\cdot, \mathbb{R}^{d}\right)$ is a bounded function by Assumption 3.4, and $(\cdot, h)$ and $\left(\cdot,{ }^{\gamma} A h\right)$ are finite (signed) measures, for $h \in \mathcal{C}_{\mathcal{K}}^{2}$. Taking averages $\frac{1}{T^{\prime}} \int_{0}^{T^{\prime}} \mathrm{d} T \ldots$ over the terms in the last equality, we get as $T^{\prime} \rightarrow \infty$

$$
\left(\gamma^{\leftarrow} R^{\leftarrow} g,{ }^{\gamma} A h\right)=-(g, h), \quad g \in \mathcal{C}_{b}^{2}, h \in \mathcal{C}_{\mathcal{K}}^{2} .
$$

5. Now consider in (50) functions $g \in \mathcal{C}_{b}^{2}$ which in addition belong to $L^{1}(\lambda)$. Then (50) can be extended from functions $h \in \mathcal{C}_{\mathcal{K}}^{2}$ to functions $h \in \mathcal{C}_{b}^{2}$. We have proved (45).

From Lemma 4.2, we deduce Theorem 3.5 and Lemma 3.6.

4.3. Proof of Theorem 3.5. Under Assumptions 3.1, 3.2, 3.4, consider an immigration measure $\pi$ having Lebesgue density $g$ in class $\mathcal{G}$. Define

$$
\tilde{m}(\mathrm{~d} v):=C\left(\gamma^{\leftarrow} R^{\leftarrow} g\right)(v) \mathrm{d} v, \quad g=\frac{\mathrm{d} \pi}{\mathrm{d} \lambda} .
$$

Then the function $\gamma^{\leftarrow} R^{\leftarrow} g$ is nonnegative, and in $L^{1}(\lambda)$ by definition of the class $\mathcal{G}$ in (34). Hence $\tilde{m}$ is a finite measure on $\mathbb{R}^{d}$. Lemma 4.2 shows that $\tilde{m}$ is a solution to Eq. (10):

$$
\left.\tilde{m}\left({ }^{\gamma} A f\right)=C{ }^{\gamma} R^{\leftarrow} g,{ }^{\gamma} A f\right)=-C(g, f)=-C \pi(f), \quad f \in \mathcal{C}_{b}^{2} .
$$

By Proposition 4.1, there is only one solution to (10) in the class of all finite measures. From 1.6+ 1.7, the invariant occupation measure $\bar{m}=C \pi^{\gamma} R$ solves (10), hence $\tilde{m}$ equals $\bar{m}$.

By Assumption 3.4, the Lebesgue density of $\tilde{m}=\bar{m}$ is a bounded function on $\mathbb{R}^{d}$.

4.4. Proof of Lemma 3.6. Under Assumptions 3.1, 3.2, 3.4, we apply Lemma 4.2 to $g \in \mathcal{C}_{b}^{2} \cap L^{1}(\lambda)$ nonnegative, and to the constant function $f=\mathbb{1}$ : then $(g, f)$ is nonnegative and finite, and

$$
(g, \mathbb{1})=\left(\gamma^{\leftarrow} R^{\leftarrow} g,-\gamma^{\gamma} A \mathbb{1}\right)=\left(\gamma^{\leftarrow} R^{\leftarrow} g, \gamma\right) \geqslant \inf _{v \in \mathbb{R}^{d}} \gamma(v) \int_{\mathbb{R}^{d}} \mathrm{~d} v^{\gamma^{\leftarrow}} R^{\leftarrow} g(v) .
$$

If $\gamma(\cdot)$ is bounded away from 0 on $\mathbb{R}^{d}$, assertion (a) of Lemma 3.6 follows.

If $\gamma^{\leftarrow}(\cdot)$ is bounded away from 0 on $\mathbb{R}^{d}$, then we have dominated convergence in

$$
v \longrightarrow \gamma^{\leftarrow} R^{\leftarrow} g(v)=E_{v}\left(\int_{0}^{\infty} \mathrm{d} t g\left(\xi_{t}^{\leftarrow}\right) \mathrm{e}^{-\int_{0}^{t} \mathrm{~d} s \gamma^{\leftarrow}\left(\xi_{s}^{\leftarrow}\right)}\right), \quad g \in \mathcal{C}_{b}^{2},
$$

( $\xi^{\leftarrow}$ is a stochastic flow of $\mathcal{C}^{2}$-diffeomorphisms), thus $\gamma^{\leftarrow} R^{\leftarrow} g$ is continuous: this is (b).

Now all results in Subsection 3.1 are proved. We turn to the results of Subsection 3.2. In order to prove Theorem 3.9, we quote an analytical result from Cattiaux [2] which - thanks to symmetry of our assumptions - we apply to both $\xi, \gamma$ and $\xi \leftarrow, \gamma^{\leftarrow}$. 
4.5. Theorem (Cattiaux [2, Proposition 2.19, Remarques 2.21]). Assume 3.8, 3.2, 3.4.

(a) One has $\gamma^{\leftarrow} R^{\leftarrow} g \in \mathcal{C}^{\infty} \cap \mathcal{S}^{\prime}$ and ${ }^{\gamma} R g \in \mathcal{C}^{\infty} \cap \mathcal{S}^{\prime}$ for $g \in \mathcal{C}^{\infty} \cap \mathcal{S}^{\prime}$.

(b) For every $h \in \mathcal{C}^{\infty} \cap \mathcal{S}^{\prime}$, there is a unique $f \in \mathcal{C}^{\infty} \cap \mathcal{S}^{\prime}$ such that $h=\gamma^{\leftarrow} A^{\leftarrow} f$.

(c) For every $h \in \mathcal{C}^{\infty} \cap \mathcal{S}^{\prime}$, there is a unique $f \in \mathcal{C}^{\infty} \cap \mathcal{S}^{\prime}$ such that $h=\gamma A f$.

(d) For every $g \in \mathcal{C}^{\infty} \cap \mathcal{S}^{\prime}$, we have $\gamma^{\leftarrow} A^{\leftarrow}\left(\gamma^{\leftarrow} R^{\leftarrow} g\right)=\gamma^{\leftarrow} R^{\leftarrow}\left(\gamma^{\leftarrow} A^{\leftarrow} g\right)=-g$.

(e) For every $f \in \mathcal{C}^{\infty} \cap \mathcal{S}^{\prime}$, we have ${ }^{\gamma} A\left({ }^{\gamma} R f\right)={ }^{\gamma} R\left({ }^{\gamma} A f\right)=-f$.

Here $\mathcal{S}^{\prime}$ is the topological dual of the Schwartz space $\mathcal{S}$ in 3.9.

4.6. Proof of Theorem 3.9. Assume 3.8, 3.2, 3.4.

1. Applying 4.5(c) to the constant function $h=\mathbb{1}$, there is a unique solution $f \in \mathcal{C}^{\infty} \cap \mathcal{S}^{\prime}$ to the equation $\gamma_{A} f=\mathbb{1}$. By 4.5(e), this solution is the function $f=-{ }^{\gamma} R \mathbb{1}=-\gamma^{\gamma} R\left(\cdot, \mathbb{R}^{d}\right.$ ) which (up to the sign) has been considered in Assumption 3.2 (and in Lemma 1.4). In virtue of this assumption, $f$ is bounded and nonpositive.

2. Consider an immigration density $g=\frac{\mathrm{d} \pi}{\mathrm{d} \lambda}$ in $\mathcal{C}_{b}^{\infty}$. For the function $f$ considered in $(1),-(g, f)$ is nonnegative and finite. Applying 4.5(d), 4.5(a) and (49), we get

$$
-(g, f)=\left(\gamma^{\leftarrow} A^{\leftarrow}\left(\gamma^{\leftarrow} R^{\leftarrow} g\right), f\right)=\left(\gamma^{\leftarrow} R^{\leftarrow} g,{ }^{\gamma} A f\right)=\left(\gamma^{\leftarrow} R^{\leftarrow} g, \mathbb{1}\right) .
$$

This shows that the $\mathcal{C}^{\infty}$-function $\gamma^{\leftarrow} R^{\leftarrow} g$ is in $L^{1}(\lambda)$.

We have proved that every immigration density $g \in \mathcal{C}_{b}^{\infty}$ belongs to class $\mathcal{G}$ considered in Theorem 3.5. Hence by 3.5 combined with 4.5 (a), the invariant occupation measure $\bar{m}=C \pi^{\gamma} R$ is Lebesgue-absolutely continuous with $\mathcal{C}^{\infty}$-density $C^{\gamma^{\leftarrow}} R^{\leftarrow}$ g. This is the first assertion of 3.9.

3. Assume that the immigration density $g=\frac{\mathrm{d} \pi}{\mathrm{d} \lambda}$ is in the Schwartz space $\mathcal{S}$. For every $h \in \mathcal{C}^{\infty} \cap \mathcal{S}^{\prime}$, we have $\gamma_{R h} \in \mathcal{C}^{\infty} \cap \mathcal{S}^{\prime}$ and $\gamma^{\leftarrow} R^{\leftarrow} g \in \mathcal{C}^{\infty} \cap \mathcal{S}^{\prime}$ by 4.5(a), and $\left(g,{ }^{\gamma} R h\right)$ is well defined and finite. Hence (49) combined with $4.5(\mathrm{~d})$ gives the duality

$$
\left(g,{ }^{\gamma} R h\right)=-\left(\gamma^{\leftarrow} A \leftarrow\left(\gamma^{\leftarrow} R^{\leftarrow} g\right),{ }^{\gamma} R h\right)=\left(\gamma^{\leftarrow} R^{\leftarrow} g,-{ }^{\gamma} A\left({ }^{\gamma} R h\right)\right)=\left(\gamma^{\leftarrow} R^{\leftarrow} g, h\right) .
$$

In particular, polynomials $h$ on $\mathbb{R}^{d}$ of arbitrary degree are elements of $\mathcal{C}^{\infty} \cap \mathcal{S}^{\prime}$, so (51) shows that $\int_{\mathbb{R}^{d}} \bar{m}(\mathrm{~d} v) h(v)$ is finite. This is the second assertion of Theorem 3.9.

4.7. Remark. (a) Assume 3.8, 3.2, 3.4, consider immigration densities in the Schwartz space $\mathcal{S}$.

By Theorem 1.6, the invariant occupation measure is given by

$$
h \rightarrow \bar{m}(h)=C \pi^{\gamma} R h=C\left(g,{ }^{\gamma} R h\right), \quad h \in \mathcal{C}^{\infty} \cap \mathcal{S}^{\prime} .
$$

Then (49) combined with Theorem 4.5 yield the strong duality appearing in (51)

$$
\left({ }^{\gamma} R\right)^{*}={ }^{\kappa} R^{\leftarrow}
$$

which identifies the Lebesgue density $\frac{\mathrm{d} \bar{m}}{\mathrm{~d} \lambda}$ as

$$
\frac{\mathrm{d} \bar{m}}{\mathrm{~d} \lambda}=C^{\gamma^{\leftarrow}} R^{\leftarrow} g .
$$

Hence Theorem 4.5 and (49) + (51) allow to pass directly from Theorem 1.6 to Theorem 3.8.

(b) This direct approach is not possible in the $\mathcal{C}^{2}$-setting of Subsection 3.1:

in the $\mathcal{C}^{2}$ context of 3.5 +3.6, or of 4.1-4.4, ${ }^{\leftarrow} R^{\leftarrow} g$ or ${ }^{\gamma} R h$ are in general not in $\mathcal{C}^{2}$, and there is no analogue of 4.5(b)-(e) for $\mathcal{C}^{2}$-functions $g, h$.

(c) The strong assumptions needed for (a) - like (37) contained in 3.8 - are not always desirable from a modelling point of view, or from the point of view of statistical inference: they exclude some simple and important models, e.g. the Ornstein-Uhlenbeck Example 3.7. 


\section{Acknowledgement}

This paper would not have been written without a fruitful series of discussions with Vlad Bally on use of Malliavin Calculus in the context of branching diffusions with immigration, opening the way to consideration of resolvants for particle motion run forwards and backwards in time. This is gratefully acknowledged.

\section{References}

[1] R. Bass, Diffusions and Elliptic Operators, Springer, 1998.

[2] P. Cattiaux, Calcul stochastique et opérateurs dégénérés du second ordre I. Résolvantes, théorème de Hörmander et applications, Bull. Sci. Math. 114 (1990) 421-462.

[3] C. Dellacherie, P.A. Meyer, Probabilités et potentiel, Hermann, Paris, 1987, Chapitres XII à XVI.

[4] R. Edwards, Functional Analysis. Theory and Applications, Holt Rinehart and Winston, 1965.

[5] R. Höpfner, E. Löcherbach, Limit Theorems for Null Recurrent Markov Processes, Mem. Amer. Math. Soc. 768 (2003).

[6] R. Höpfner, M. Hoffmann, E. Löcherbach, Non-parametric estimation of the death rate in branching diffusions, Scand. J. Statist. 29 (2002) $665-692$.

[7] L. Hörmander, Hypoelliptic second-order differential equations, Acta Math. 119 (1967) 147-171.

[8] I. Ibragimov, R. Has'minskii, Statistical Estimation, Springer, 1981.

[9] N. Ikeda, S. Watanabe, Stochastic Differential Equations and Diffusion Processes, North Holland-Kodansha, 1989.

[10] H. Kunita, Stochastic differential equations and stochastic flow of diffeomorhisms, in: P. Hennequin (Ed.), École d'été de probabilités de St. Flour XII, 1982, in: Lecture Notes in Math., vol. 1097, Springer, 1984, pp. 143-304.

[11] H. Kunita, Stochastic Flows and Stochastic Differential Equations, Cambridge University Press, 1990.

[12] S. Kusuoka, D. Stroock, Applications of the Malliavin Calculus, part II, J. Fac. Sci. Univ. Tokyo Sect. IA Math. 32 (1985) 1-76.

[13] L. Le Cam, G. Yang, Asymptotic in Statistics, Springer, 1990.

[14] E. Löcherbach, Likelihood ratio processes for Markovian particle systems with killing and jumps, Statist. Inference Stoch. Process. 5 (2002) 153-177.

[15] E. Löcherbach, LAN and LAMN for systems of interacting diffusions with branching and immigration, Ann. Inst. H. Poincaré Probab. Statist. 38 (2002) 59-90.

[16] E. Löcherbach, Smoothness of the intensity measure density for interacting branching diffusions with immigrations, J. Funct. Anal. 215 (2004) 130-177.

[17] D. Revuz, Markov Chains, North-Holland, 1975.

[18] D. Williams, To begin at the beginning: ..., in: Stochastic Integrals (Proc. Durham 1980), in: Lecture Notes in Math., vol. 851, Springer, 1981, pp. 1-55.

[19] A.M. Zubkov, Life-periods of a branching process with immigration, Theor. Probab. Appl. 17 (1972) 174-183. 